Pacific

Journal of

Mathematics

BESSEL FUNCTIONS FOR GL(3) OVER A $p$-ADIC FIELD

Ehud Moshe Baruch

Volume $211 \quad$ No. 1

September 2003 


\title{
BESSEL FUNCTIONS FOR GL(3) OVER A $p$-ADIC FIELD
}

\author{
Ehud Moshe Baruch
}

We attach Bessel functions to generic representations of $\mathrm{GL}(3, k)$ where $k$ is a $p$-adic field and study their asymptotics.

\section{Introduction and main results.}

Let $k$ be a non-archimedean local field. Bessel functions for the double cover of GL $(2, k)$ were constructed by Gelbart and Piatetski-Shapiro in [G-PS] and used to compute some epsilon factors. A more detailed analysis of the Bessel functions for GL $(2, k)$ was obtained by Soudry in $[\mathbf{S}]$ and again used to compute some epsilon factors. Bessel functions for $\operatorname{GL}(2, \mathbf{R})$ were defined and analyzed by Cogdell and Piatetski-Shapiro in [C-PS1]. Bessel functions were also discussed in Averbuch's thesis $[\mathbf{A v}]$. In $[\mathbf{B a}]$ we attached Bessel functions to every generic representation of a quasi-split reductive group over $k$ using a distribution approach similar to Harish-Chandra's approach for the character functions. In the present paper we attach Bessel functions to generic representations of $\mathrm{GL}(3, k)$ using a different method and study their asymptotics. In $[\mathbf{B a 2}]$ we use the results of this paper to show that both approaches yield the same Bessel functions.

1.1. Main results. We state here our main theorems. We shall only consider here the main Bessel function of a representation which is the one attached to the open Bruhat cell. Other Bessel functions are described in Section 6.

Let $G=\mathrm{GL}(3, k)$ and let $B$ be the Borel subgroup of upper triangular matrices, $A$ the subgrooup of diagonal matrices and $N$ the subgroup of upper unipotent matrices. Let $\psi$ be a nondegenerate character of $N$. Let $\mathbb{W}=N(A) / A$ be the Weyl group where $N(A)$ is the normalizer of $A$. We identify $\mathbb{W}$ with the set of six permutation matrices in $N(A)$. Let

$$
w_{0}=\left(\begin{array}{lll} 
& & 1 \\
& 1 & \\
1 & &
\end{array}\right)
$$

be the longest Weyl element in $\mathbb{W}$. Let $(\pi, V)$ be an irreducible admissible representation of $G$. We say that $\pi$ is generic if there exist a nonzero functional $L: V \rightarrow C$ such that

$$
L(\pi(n) v)=\psi(n) L(v) \quad n \in N, v \in V .
$$


It is well-known that such a functional is unique up to scalar multiples. We call this functional a $\psi$ Whittaker functional. Now define

$$
W_{v}(g)=L(\pi(g) v) \quad v \in V, g \in G,
$$

and let $\mathrm{G}$ act on the space of these functions by right translations. Then the map $v \rightarrow W_{v}$ gives a realization of $\pi$ on a space of Whittaker functions satisfying

$$
W_{v}(n g)=\psi(n) W_{v}(g) \quad n \in N, g \in G .
$$

We denote this space by $\mathcal{W}(\pi, \psi)$. In Section 4 we define the subspace $\mathcal{W}^{0}(\pi, \psi)$ of $\mathcal{W}(\pi, \psi)$. In the case where $\pi$ is supercuspidal we have that $\mathcal{W}^{0}(\pi, \psi)=\mathcal{W}(\pi, \psi)$. Let $\alpha_{1}, \alpha_{2}$ be the positive roots realized as functions on $A$ (See (2.9)). Let $M>0$ be a constant and let

$$
A^{M}=A^{M}\left(w_{0}\right)=\left\{a \in A: \alpha_{i}(a)<M, i=1,2\right\} .
$$

Our first main theorem is the following:

Theorem 1.1. Let $W \in \mathcal{W}^{0}(\pi, \psi)$ and $M$ a positive constant. Then the function

$$
(a, n) \mapsto W\left(a w_{0} n\right)
$$

defined on the set $A^{M} \times N$ is compactly supported in $N$. That is, if $W\left(a w_{0} n\right)$ $\neq 0$ and $a \in A^{M}, n \in N$ then $n$ is in some compact set independent of $a$.

Since $A^{M}$ cover $A$ as $M \rightarrow \infty$ we get the following Corollary:

Corollary 1.2. Let $\pi$ be a supercuspidal representation of $G$ and $W \in$ $\mathcal{W}(\pi, \psi)$ a Whittaker function associated to $\pi$. Fix $g \in B w_{0} B$. Then the function

$$
n \mapsto W(g n)
$$

is compactly supported in $N$.

Proof. Write $g=n_{1} a w_{0} n_{2}$ and choose $M$ large enough such that $a \in A^{M}$. Since $W(g n)=\psi\left(n_{1}\right) W\left(a w_{0} n_{2} n\right)$ the result follows from Theorem 1.1.

This result allows us to define Bessel functions for supercuspidal representations. In order to treat all irreducible admissible representations we will need the following result which allows us to move from $\mathcal{W}(\pi, \psi)$ to $\mathcal{W}^{0}(\pi, \psi)$.

Theorem 1.3. Let $W \in \mathcal{W}(\pi, \psi)$. There exist a compact open subgroup $N_{0}=N_{0}(W)$ of $N$ such that the function $W_{N_{0}, \psi} \in \mathcal{W}^{0}(\pi, \psi)$.

Here $W_{N_{0}, \psi}$ is defined by

$$
W_{N_{0}, \psi}(g)=\int_{N_{0}} W(g n) \psi^{-1}(n) d n .
$$

Corollary 1.4. $\mathcal{W}^{0}(\pi, \psi) \neq\{0\}$.

Proof. Let $W \in \mathcal{W}(\pi, \psi)$ be such that $W(e) \neq 0$. Then $W_{N_{0}, \psi}(e) \neq 0$ for every compact open subgroup $N_{0}$ in $N$. 
Let $N_{1} \subset N_{2} \subset N_{3} \subset \ldots$ be a filtration of $N$ with compact open subgroups $N_{i}, i=1,2, \ldots$, such that $N=\bigcup_{i=1}^{\infty} N_{i}$. We denote this filtration by $\mathcal{N}$. Let $f: N \rightarrow \mathbb{C}$ be a locally constant function.

\section{Definition 1.5.}

$$
\int_{N}^{\mathcal{N}} f(n) d n=\lim _{m \rightarrow \infty} \int_{N_{m}} f(n) d n
$$

if this limit exists.

Corollary 1.6. Let $\mathcal{N}=\left\{N_{i}, i>0\right\}$ be a filtration of $N$ as above. Let $g \in B w_{0} B$ and $W \in \mathcal{W}(\pi, \psi)$. Then

$$
\int_{N}^{\mathcal{N}} W(g n) \psi^{-1}(n) d n
$$

is convergent, and the value is independent of the choice of filtration $\mathcal{N}$.

Proof. Let $N_{0}=N_{0}(W)$ be a compact open subgroup of $N$ as in Theorem 1.3. There exist an integer $M$ such that $N_{0} \subset N_{m}$ for all $m>M$. Let $m>M$. We have

$$
\begin{aligned}
& \frac{1}{\operatorname{vol}\left(N_{0}\right)} \int_{N_{m}} W_{N_{0}, \psi}(g n) \psi^{-1}(n) d n \\
& =\frac{1}{\operatorname{vol}\left(N_{0}\right)} \int_{N_{m}} \int_{N_{0}} W\left(g n_{1} n_{2}\right) \psi^{-1}\left(n_{1} n_{2}\right) d n_{1} d n_{2} .
\end{aligned}
$$

Applying Fubini and changing variables $n=n_{1} n_{2}$ we get that the last integral is the same as

$$
\int_{N_{m}} W(g n) \psi^{-1}(n) d n
$$

By Theorem 1.1 the function $n \mapsto W_{N_{0}, \psi}(g n)$ is compactly supported in $N$, hence we can take the limit $m \rightarrow \infty$ in (1.3) to get the value

$$
\frac{1}{\operatorname{vol}\left(N_{0}\right)} \int_{N} W_{N_{0}, \psi}(g n) \psi^{-1}(n) d n \text {. }
$$

It is clear that this value is independent of the filtration $\mathcal{N}$.

Let $g \in B w_{0} B$ and define the linear functional $L_{g}: V \rightarrow \mathbb{C}$ by

$$
L_{g}(v)=\int_{N}^{\mathcal{N}} W_{v}(g n) \psi^{-1}(n) d n
$$

It is easy to see that $L_{g}$ is a Whittaker functional, hence it follows from the uniqueness of Whittaker functionals that there exist a scalar $j_{\pi, \psi}(g)$ such that

$$
L_{g}(v)=j_{\pi, \psi}(g) L(v)
$$


for all $v \in V$. We call $j_{\pi}=j_{\pi, \psi}$ the Bessel function of $\pi$. (See Section 6 for other Bessel functions.) The Bessel function $j_{\pi}$ is defined on the set $B w_{0} B$ and we will prove that it is locally constant there. It is clear that $j_{\pi}(g)$ satisfies

$$
j_{\pi}\left(n_{1} g n_{2}\right)=\psi\left(n_{1} n_{2}\right) j_{\pi}(g), \quad n_{1}, n_{2} \in N, g \in B w_{0} B,
$$

hence it is determined by its values on the set $A w_{0}$. As is the case with the character of the representation $[\mathbf{H C}]$, the Bessel function $j_{\pi}$ is expected to be locally integrable on $G$. Harish-Chandra's proof of the local integrability of the character depended on certain relations between the asymptotics of the character and certain orbital integrals. In this paper we establish that the asymptotics of $j_{\pi}$ are the same as the asymptotics of certain orbital integrals which were studied by Jacquet and Ye [J-Y1]. This result will allow us to show in $[\mathbf{B a 2}]$ that $j_{\pi}$ is locally integrable and gives the Bessel distribution on $G$. We now describe the relation between the Bessel functions and orbital integrals.

Let $C_{c}^{\infty}(G)$ be the space of locally constant and compactly supported functions on $G$. Let $Z$ be the center of $G$ and let $\omega$ be a quasi character on $Z$. (6))

For $\phi \in C_{c}^{\infty}(G)$ and $g \in B w_{0} B$ we define the orbital integral (see [J-Y1]

$$
J_{\psi, \omega}(g, \phi)=\int_{N \times Z \times N} \phi\left(n_{1} z g n_{2}\right) \psi^{-1}\left(n_{1} n_{2}\right) \omega^{-1}(z) d n_{1} d n_{2} d z .
$$

It follows from $[\mathbf{J}-\mathbf{Y} \mathbf{1}]$ that this integral converges absolutely and defines a locally constant function on $B w_{0} B$.

Theorem 1.7. Let $\pi$ be an irreducible admissible representation of $G$ with central character $\omega_{\pi}$. Let $x \in G$. There exist a neighborhood $U_{x}$ of $x$ in $G$ and a function $\phi \in C_{c}^{\infty}(G)$ such that

$$
j_{\pi, \psi}(g)=J_{\psi, \omega_{\pi}}(g, \phi)
$$

for all $g \in U_{x}$.

Remark 1.8. Since $j_{\pi, \psi}$ and $J_{\psi, \omega_{\pi}}$ are only defined on $B w_{0} B$ we are really asserting the equality on $B w_{0} B \cap U_{x}$. Another option is to define these functions as having value zero outside of $B w_{0} B$ in which case the equality above does hold. In any case, the equality is true up to a set of measure zero.

Corollary 1.9. If $g \mapsto J_{\psi, \omega_{\pi}}(g, \phi)$ is locally integrable as a function on $G$ for every $\phi \in C_{c}^{\infty}(G)$ then $j_{\pi, \psi}$ is locally integrable on $G$.

Hence the question of local integrability of the Bessel function reduces to the question of the local integrability of the orbital integral. As mentioned above, we shall consider this question in [Ba2]. 
Our paper is divided as follows: In Section 2 we introduce some notations and study certain root and weight spaces. In Section 3 we describe the method of proof used for our main results and prove a result which is needed later using this method. In Section 4 we prove a more general version of Theorem 1.1. In Section 5 we prove Theorem 1.3. In Section 6 we define Bessel functions including Bessel functions attached to other Weyl elements. In Section 7 we prove Theorem 1.7.

\section{Notations and preliminaries.}

Let $k$ be a non-archimedean local field. Let $O$ be the ring of integers in $k$ and let $P$ be the maximal ideal in $k$. Let $\varpi$ be a generator of $P$. We denote by $|x|$ the normalized absolute value of $x \in k$. Let $q=|O / P|$ be the order of the residue field of $k$. Then $|\varpi|=q^{-1}$. Let $G=\mathrm{GL}(3, k)$ and let $A$ be the group of diagonal matrices in $G$ consisting of matrices of the form

$$
d\left(a_{1}, a_{2}, a_{3}\right)=\left(\begin{array}{lll}
a_{1} & & \\
& a_{2} & \\
& & a_{3}
\end{array}\right) .
$$

We let

$$
Z=Z(G)=\left\{d(a, a, a): a \in k^{*}\right\} .
$$

Let $X(A)=\operatorname{Hom}_{k}(A, k)$ be the group of rational homomorphisms. Then each $\alpha \in X(A)$ is of the form

$$
\alpha\left(d\left(a_{1}, a_{2}, a_{3}\right)\right)=a_{1}^{n_{1}} a_{2}^{n_{2}} a_{3}^{n_{3}}
$$

with $n_{1}, n_{2}, n_{3} \in \mathbf{Z}$. We view $X(A)$ as a group under addition where the addition is defined by

$$
(\alpha+\beta)(a)=\alpha(a) \beta(a), \quad \alpha, \beta \in X(A), a \in A .
$$

We let $|X|=X(A) \otimes \mathbf{R}$. Then we shall identify $|X|$ with the vector space of functions $|\alpha|=|\alpha|_{\lambda_{1}, \lambda_{2}, \lambda_{3}}$ from $A$ to $\mathbf{R}$ of the form

$$
|\alpha|\left(d\left(a_{1}, a_{2}, a_{3}\right)\right)=\left|a_{1}\right|^{\lambda_{1}}\left|a_{2}\right|^{\lambda_{2}}\left|a_{3}\right|^{\lambda_{3}}
$$

where $\lambda_{1}, \lambda_{2}, \lambda_{3} \in \mathbf{R}$. Here addition is defined as in (2.2) and scalar multiplication is defined by

$$
(\lambda|\alpha|)(a)=(|\alpha|(a))^{\lambda}, \quad|\alpha| \in|X|, a \in A, \lambda \in \mathbf{R} .
$$

We define an inner product on $|X|$ by

$$
\left\langle\alpha_{\lambda_{1}, \lambda_{2}, \lambda_{3}}, \alpha_{\gamma_{1}, \gamma_{2}, \gamma_{3}}\right\rangle=\sum_{i=1}^{3} \lambda_{i} \gamma_{i} .
$$


For $i, j \in\{1,2,3\}, i \neq j$ we let $\alpha_{i, j}: A \rightarrow k$ be the functions defined by

$$
\alpha_{i, j}\left(d\left(a_{1}, a_{2}, a_{3}\right)\right)=\frac{a_{i}}{a_{j}}
$$

and $|\alpha|_{i, j}(a)=\left|\alpha_{i, j}(a)\right|$. Let $\Phi=\left\{\alpha_{i, j}\right\}$. Then $|\Phi|=\left\{|\alpha|_{i, j}\right\}$ is a root system in $|X|$. We have that $\Phi=\Phi^{+} \cup \Phi^{-}$where $\Phi^{+}=\left\{\alpha_{i, j}: i<j\right\}$ is the set of positive roots and $\Phi^{-}$is the set of negative roots. Let $E_{i, j}$ be the matrix whose $(i, j)$ th entry is 1 and all other entries are zero. For $\alpha=\alpha_{i, j} \in \Phi$ and for $b \in k$ we let

$$
\begin{aligned}
& x_{\alpha}(b)=x_{i, j}(b)=I+b E_{i, j} \\
& h_{\alpha}(b)=h_{i, j}(b)=b E_{i, i}-b^{-1} E_{j, j} .
\end{aligned}
$$

For each $\alpha \in \Phi$ we let $N_{\alpha}=\left\{x_{\alpha}(b): b \in k\right\}$. Let $\mathbb{W}=N(A) / A$ be the Weyl group of $G$. We shall identify $\mathbb{W}$ with $S_{3}$, the symmetric group. In particular if $\sigma \in S_{3}$ then we let $w_{\sigma}$ be the associated permutation matrix. For every $\alpha=\alpha_{i, j} \in \Phi$ we set $w_{\alpha}=w_{(i, j)}$ where $(i, j)$ is a transposition in $S_{3}$. W acts on $\Phi$ and $|\Phi|$ in a natural way. We have that if $i \neq j$ then

$$
\begin{gathered}
a x_{i, j}(b) a^{-1}=x_{i, j}\left(\alpha_{i, j}(a) b\right), \quad a \in A, b \in k \\
x_{i, j}(b) x_{j, i}\left(-b^{-1}\right) x_{i, j}(b)=w_{(i, j)} h_{j, i}(b), \quad b \in k .
\end{gathered}
$$

Let $N$ be the subgroup of upper unipotent matrices. Then every $n \in N$ can be written uniquely in the form

$$
n=x_{1,3}\left(b_{1}\right) x_{1,2}\left(b_{2}\right) x_{1,3}\left(b_{3}\right)
$$

where $b_{1}, b_{2}, b_{3} \in k$.

2.1. Roots, weights and Weyl elements. The root system $|\Phi|$ spans a subspace $|V|$ of $|X|$ given by

$$
|V|=\left\{|\alpha|_{\lambda_{1}, \lambda_{2}, \lambda_{3}}: \lambda_{1}+\lambda_{2}+\lambda_{3}=0\right\} .
$$

Then $\Delta=\left\{|\alpha|_{1,2},|\alpha|_{2,3}\right\}$ is a basis for $|V|$ consisting of simple roots. If $\mathcal{B}$ is a basis for $|V|$ then we denote by $\mathcal{B}^{*}$ the dual basis (up to scalars) with respect to (2.4). In particular, the fundamental weights $\lambda_{1}, \lambda_{2}$ give the basis dual to $\Delta$ where

$$
\lambda_{1}=|\alpha|_{2,-1,-1}, \quad \lambda_{2}=|\alpha|_{1,1,-2} .
$$

We write $\Delta=\left\{\alpha_{1}, \alpha_{2}\right\}$ and $\Delta^{*}=\left\{\lambda_{1}, \lambda_{2}\right\}$ with

$$
\alpha_{1}=|\alpha|_{1,2}=|\alpha|_{1,-1,0}, \quad \alpha_{2}=|\alpha|_{2,3}=|\alpha|_{0,1,-1}
$$

and $\lambda_{1}, \lambda_{2}$ as in (2.8). If $S \subseteq \Delta$ then we let

$$
\mathcal{B}(S)=\left\{\gamma_{1}, \gamma_{2}\right\}
$$


where $\gamma_{i}$ is defined by

$$
\gamma_{i}= \begin{cases}\alpha_{i} & \text { if } \alpha_{i} \in S ; \\ \lambda_{i} & \text { if } \alpha_{i} \notin S .\end{cases}
$$

We let $\mathcal{B}^{*}(S)=(\mathcal{B}(S))^{*}$ (up to scalars). We shall fix the following sets:

$$
\begin{array}{lll}
\mathcal{B}(\emptyset)=\Delta^{*}, & \mathcal{B}\left(\left\{\alpha_{1}\right\}\right)=\left\{\alpha_{1}, \lambda_{2}\right\}, \quad \mathcal{B}\left(\left\{\alpha_{2}\right\}\right)=\left\{\lambda_{1}, \alpha_{2}\right\}, & \mathcal{B}(\Delta)=\Delta \\
\mathcal{B}^{*}(\emptyset)=\Delta, & \mathcal{B}^{*}\left(\left\{\alpha_{1}\right\}\right)=\left\{\alpha_{1}, \lambda_{2}\right\}, \mathcal{B}^{*}\left(\left\{\alpha_{2}\right\}\right)=\left\{\lambda_{1}, \alpha_{2}\right\}, & \mathcal{B}^{*}(\Delta)=\Delta^{*}
\end{array}
$$

For each $\alpha \in \Phi$ we let $w_{\alpha} \in \mathbb{W}$ be the reflection associated with $\alpha$. In particular, $w_{\alpha_{1,2}}=w_{(1,2)}$ and $w_{\alpha_{2,3}}=w_{(2,3)}$. Since $\mathbb{W}$ is generated by $w_{(1,2)}$ and $w_{(2,3)}$ it follows that each $w$ can be written as a product of these transpositions. We define $S(w) \subset \Delta$ to be

$$
S(w)=\left\{\beta_{1}, \ldots, \beta_{k}\right\}
$$

where $w=w_{\beta_{1}} \cdots w_{\beta_{k}}$ is a minimal decomposition of $w$ into a product of such transpositions. In particular we have

$$
\begin{gathered}
S(e)=\emptyset, \quad S((1,2))=\left\{\alpha_{1,2}\right\}, \quad S((2,3))=\left\{\alpha_{2,3}\right\} \\
S((1,2,3))=\Delta, \quad S((1,3,2))=\Delta, \quad S((1,3))=\Delta .
\end{gathered}
$$

It is well-known that $S(w)$ is independent of the minimal decomposition. We define

$$
S^{-}(w)=\left\{\alpha \in \Phi^{+}: w(\alpha)<0\right\}, \quad S^{+}(w)=\left\{\alpha \in \Phi^{+}: w(\alpha)>0\right\}
$$

and

$$
N_{w}^{-}=\prod_{\alpha \in S^{-}(w)} N_{\alpha}, \quad N_{w}^{+}=\prod_{\alpha \in S^{+}(w)} N_{\alpha} .
$$

Then $N=N_{w}^{+} N_{w}^{-}$.

\section{Method of proof.}

The main method of proof in this paper is a careful analysis of the relation between the Bruhat Decomposition and the Iwasawa decomposition of our group. Such relations were explored by previous authors such as $[\mathbf{B o}-\mathbf{H C}]$, [Bo], [J-PS-S]. In this paper we present an explicit method of obtaining such information which follows a simple pattern. The idea is to analyze the Bruhat cells inductively going from the closed cell up to the open cell. The induction is on the length of the respective Weyl element. Another induction takes place inside an individual cell where we "peel" the root groups one by one. We find it very striking that the three main results in this paper were all proved using this method. In this section we present a new proof for a well-known result which demonstrates best how the method works.

Since we have only four different lengths for the Weyl elements of GL(3) we shall not employ induction in this paper. The induction process will be 
used in a future paper on GL $(n)$. Before we go to the main result of this section we shall indicate how the method works.

3.1. Example. Let

$$
w=w_{(1,2,3)}=\left(\begin{array}{lll}
0 & 0 & 1 \\
1 & 0 & 0 \\
0 & 1 & 0
\end{array}\right) .
$$

Then every $g \in B w B$ can be written uniquely in the form

$$
g=\operatorname{nawx}_{1,3}\left(b_{1}\right) x_{2,3}\left(b_{2}\right),
$$

with $n \in N, a \in A$ and $b_{1}, b_{2} \in k$. Our "peeling" proceeds in the following way: If we have a function $F$ which is smooth on the right then for large enough $\left|b_{2}\right|$ we have that $F\left(g x_{3,2}\left(-b_{2}^{-1}\right)\right)=F(g)$ for all $g \in G$. In particular, if $g \in B w B$ is written as above then $g_{1}=g x_{3,2}\left(-b_{2}^{-1}\right) \in B w_{1} B$ with $B w_{1} B$ being a smaller cell than $B w B$, that is, the length of $w_{1}$ is less than the length of $w$. At this point we know that the values of $F$ on such $g$ for which $\left|b_{2}\right|$ is large are determined by the values of $F$ on smaller cells and we can use the induction hypothesis (whatever that may be for each proof) to conclude the result for such $b_{2}$. To continue, we need to control the values of $F$ for bounded $\left|b_{2}\right|$ which depends on the result we are trying to prove. Once this is complete we have "peeled" the root subgroup $\left\{x_{2,3}\left(b_{2}\right)\right\}$ and we are left to treat the case $g=\operatorname{nawx}_{1,3}\left(b_{1}\right)$. At this point we look at the case where $\left|b_{1}\right|$ is large and multiply $g$ by $x_{1,3}\left(-b_{1}^{-1}\right)$ to get $g_{1}=g x_{1,3}\left(-b_{1}^{-1}\right) \in B w_{2} B$ with length of $w_{2}$ less than the length of $w$. The argument continues in this way.

3.2. Iwasawa decomposition. Let $K=\mathrm{GL}(2, O)$. Then it is well-known that

$$
G=N A K \text {. }
$$

For every $|\alpha| \in|X|$ we extend $|\alpha|$ (see [J-PS-S]) to $G$ by defining

$$
|\alpha|(g)=|\alpha|(a)
$$

where $g=n a k, n \in N, a \in A, k \in K$, is an Iwasawa decomposition of $g$. It is easy to see that $|\alpha|$ is independent of the choice of decomposition.

Recall that $\Delta^{*}=\left\{\lambda_{1}, \lambda_{2}\right\}$ is the set of fundamental weights where $\lambda_{1}=$ $|\alpha|_{2,-1,-1}$ and $\lambda_{2}=|\alpha|_{1,1,-2}$ (See (2.3).) We view $\lambda_{1}$ and $\lambda_{2}$ as functions on $G$ as above. The main theorem of this section is the following:

Theorem 3.1. Let $\lambda \in \Delta^{*}$ and $w \in \mathbb{W}$. Then

$$
\lambda(w n) \leq 1
$$

for every $n \in N_{w}^{-}$. 
Proof. The proof is a case by case argument starting with the smaller Bruhat cells and moving to the larger cells.

Case 1. $w=e$.

In this case $N_{w}^{-}=\{e\}$. Hence, $w n=e$. Since $\lambda(e)=1$ we are done.

Case 2. $w=w_{(1,2)}$.

In this case $N_{w}^{-}=N_{1,2}$. Hence $w n=w x_{1,2}(b)$. If $|b| \leq 1$ then $w n \in K$ and $\lambda(w n)=1$. If $|b|>1$ then $x_{2,1}\left(-b^{-1}\right) \in K$ and

$$
\lambda(w n)=\lambda\left(w x_{1,2}(b)\right)=\lambda\left(w x_{1,2}(b) x_{2,1}\left(-b^{-1}\right)\right)=\lambda\left(h_{2,1}(b)\right) \leq 1 .
$$

Case 3. $w=w_{(2,3)}$.

This case is similar to Case 2 and is omitted.

Case $4 . \quad w=w_{(1,2,3)}$.

In this case $N_{w}^{-}=N_{1,3} N_{2,3}$. We first look at a special case where $w n=$ $w x_{1,3}\left(b_{1}\right)$. If $\left|b_{1}\right| \leq 1$ then $w n \in K$ and $\lambda(w n)=1$. If $\left|b_{1}\right|>1$ then $x_{3,1}\left(-b_{1}^{-1}\right) \in K$ and

$$
\begin{aligned}
\lambda\left(w x_{1,3}\left(b_{1}\right)\right) & =\lambda\left(w x_{1,3}\left(b_{1}\right) x_{3,1}\left(-b_{1}^{-1}\right)\right) \\
& =\lambda\left(x_{2,3}(1) h_{2,1}\left(b_{1}\right) w_{(2,3)}\right)=\lambda\left(h_{2,1}\left(b_{1}\right)\right) \leq 1 .
\end{aligned}
$$

For the general case, assume

$$
w n=w x_{1,3}\left(b_{1}\right) x_{2,3}\left(b_{2}\right) .
$$

If $\left|b_{2}\right| \leq 1$ then $x_{2,3}\left(b_{2}\right) \in K$ and $\lambda(w n)=\lambda\left(w x_{1,3}\left(b_{1}\right)\right) \leq 1$ by the special case above. If $\left|b_{2}\right|>1$ then $x_{3,2}\left(-b_{2}^{-1}\right) \in K$ and we have

$$
\lambda(w n)=\lambda\left(w x_{1,3}\left(b_{1}\right) x_{2,3}\left(b_{2}\right) x_{3,2}\left(-b_{2}^{-1}\right)\right) .
$$

Since $w x_{1,3}\left(b_{1}\right) x_{2,3}\left(b_{2}\right) x_{3,2}\left(-b_{2}^{-1}\right)=n^{\prime} h_{3,1}\left(b_{2}\right) w_{(1,2)} n^{\prime \prime}$ for some $n^{\prime} \in N$ and $n^{\prime \prime} \in N_{w_{(1,2)}}^{-}$and since $\lambda\left(n^{\prime} h_{3,1}\left(b_{2}\right) w_{(1,2)} n^{\prime \prime}\right)=\lambda\left(h_{3,1}\left(b_{2}\right)\right) \lambda\left(w_{(1,2)} n^{\prime \prime}\right)$ we can use the assumption on $\left|b_{2}\right|$ and Case 2 to conclude that $\lambda(w n) \leq 1$.

Case 5. $w=w_{(1,3,2)}$.

This case is similar to Case 4 and is omitted.

Case 6. $w=w_{(1,3)}=w_{0}$.

In this case $N_{w}^{-}=N$. We first prove two special cases. We show that $\lambda\left(w x_{1,3}\left(b_{1}\right)\right) \leq 1$ and $\lambda\left(w x_{1,3}\left(b_{1}\right) x_{2,3}\left(b_{2}\right)\right) \leq 1$. The proof is similar to Case 4 and is omitted. The general case follows the same pattern. Let $w n=w x_{1,3}\left(b_{1}\right) x_{2,3}\left(b_{2}\right) x_{1,2}\left(b_{3}\right)$. If $\left|b_{3}\right| \leq 1$ then $x_{1,2}\left(b_{3}\right) \in K$ and $\lambda(w n)=\lambda\left(w x_{1,3}\left(b_{1}\right) x_{2,3}\left(b_{2}\right)\right) \leq 1$ by the special case above. Assume $\left|b_{3}\right|>1$. Let $g^{\prime}=w n x_{2,1}\left(-b_{3}^{-1}\right)$. Then $\lambda(w n)=\lambda\left(g^{\prime}\right)$. We can write $g^{\prime}=n^{\prime} h_{3,2}\left(b_{3}\right) w_{(1,2)} n^{\prime \prime}$ for some $n^{\prime} \in N, n^{\prime \prime} \in N_{w_{(1,2,3)}}^{-}$. Hence $\lambda\left(g^{\prime}\right)=$ $\lambda\left(h_{3,2}\left(b_{3}\right)\right) \lambda\left(w_{(1,3,2)} n^{\prime \prime}\right) \leq 1$ by Case 4 and by the condition on $\left|b_{3}\right|$. 


\section{Spaces of Whittaker functions.}

In this section we define a subspace of the space of Whittaker functions on $G$ and prove some properties of this subspace. In particular we prove Theorem 4.6 which asserts that certain functions on unipotent subgroups are compactly supported. This is one of our main theorems in this paper.

4.1. Whittaker functions. Let $\psi_{k}$ be a character of $k$ and assume that $\psi_{k}$ is identically one on $O$ and nontrivial on $P^{-1}$. For a unipotent matrix $n \in N$ we set

$$
\psi(n)=\psi_{k}\left(n_{1,2}+n_{2,3}\right)
$$

where $n_{i, j}$ are the entries of $n$. Then

$$
\psi\left(x_{1,2}\left(b_{1}\right) x_{2,3}\left(b_{2}\right) x_{1,3}\left(b_{3}\right)\right)=\psi_{k}\left(b_{1}+b_{2}\right) .
$$

We let $\mathcal{W}=\mathcal{W}(G, \psi)$ be the set of functions $W: G \rightarrow \mathcal{C}$ such that $W$ is smooth on the right and

$$
W(n g)=\psi(n) W(g), \quad n \in N, g \in G .
$$

Examples of such functions are Whittaker functions associated with generic representations of $G$. Other examples are given by projecting compactly supported and locally constant functions to this space as follows:

$$
W_{f}(g)=\int_{N} f(n g) \psi^{-1}(n) d n, \quad f \in C_{c}^{\infty}(G) .
$$

We shall study the space of such functions $\left\{W_{f}: f \in C_{c}^{\infty}(G)\right\}$ in Section 7 .

For every $|\alpha| \in|X|$ we extend $|\alpha|$ to $G$ as in (3.1).

For $w \in \mathbf{W}$ we set

$$
S^{0}(w)=S\left(w w_{0}\right)
$$

where $S\left(w w_{0}\right)$ is defined in (2.12).

Definition 4.1. Let $\mathcal{W}=\mathcal{W}(G, \psi)$ be the space of Whittaker functions defined above. We define $\mathcal{W}^{0}=\mathcal{W}^{0}(G, \psi) \subset \mathcal{W}$ to be the set of functions $W \in \mathcal{W}$ such that for every $w \in \mathbf{W}$ and every $\alpha \in S^{0}(w)$ there exist positive constants $D_{\alpha}<E_{\alpha}$ such that if $g \in B w B$ then

$$
W(g) \neq 0 \Longrightarrow D_{\alpha}<|\alpha|(g)<E_{\alpha}, \quad \alpha \in S^{0}(w) .
$$

In other words, $W \in \mathcal{W}^{0}$ if for each $w \in \mathbb{W}$ and each $\alpha \in S^{0}(w)$ the support of $W$ in $B w B$ has bounded image under $\alpha$.

Remark 4.2. The condition $|\alpha|(g)<E_{\alpha}, \alpha \in S^{0}(w)$ that appears in (4.3) is redundant since by [J-PS-S] the support of $W$ is contained in the set $\{g:|\alpha|(g)<K, \alpha \in \Delta\}$ for some positive number $K$. Moreover, if $W$ is a Whittaker function in the Whittaker model of a supercuspidal representation of $G$ then it follows from [J-PS-S] that $W$ is compactly supported $\bmod N Z$. 
Hence it follows that $W$ satisfies condition (4.3) for every $\alpha \in \Delta$ and every $g \in G$ and in particular $W \in \mathcal{W}^{0}$.

Definition 4.3. Let $\alpha \in \Delta$. we define the sets

$$
\begin{aligned}
& X_{C_{1}, C_{2}}(\alpha)=\left\{g \in G\left|C_{1}<\right| \alpha \mid(g)<C_{2}\right\}, \\
& A_{C_{1}, C_{2}}(\alpha)=\left\{a \in A\left|C_{1}<\right| \alpha \mid(a)<C_{2}\right\}
\end{aligned}
$$

and the sets

$$
X_{C_{1}, C_{2}}=\bigcap_{\alpha \in \Delta} X_{C_{1}, C_{2}}(\alpha), \quad A_{C_{1}, C_{2}}=\bigcap_{\alpha \in \Delta} A_{C_{1}, C_{2}}(\alpha) .
$$

Lemma 4.4. Let $\alpha \in \Delta, C_{1}<C_{2}$ positive numbers and $R$ a compact set in $G$. Then:

(a) There exist constants $C_{1}^{\prime}<C_{2}^{\prime}$ such that

$$
X_{C_{1}, C_{2}}(\alpha) R \subset X_{C_{1}^{\prime}, C_{2}^{\prime}}(\alpha) .
$$

(b) Let $Y$ be a subset of $G$ and assume that for every $y \in Y$ there exist $r \in R$ such that $y r \in X_{C_{1}, C_{2}}(\alpha)$. Then there exist positive constants $C_{1}^{\prime}<C_{2}^{\prime}$ such that $Y \subset X_{C_{1}^{\prime}, C_{2}^{\prime}}(\alpha)$.

Proof. (a) We can write $X_{C_{1}, C_{2}}(\alpha)=N A_{C_{1}, C_{2}}(\alpha) K$. It is clear that

$$
|\alpha|\left(X_{C_{1}, C_{2}} R\right)=|\alpha|\left(A_{C_{1}, C_{2}}(\alpha)\right)|\alpha|(K R) .
$$

Since $K R$ is a compact set in $G$ and $|\alpha|$ is continuous the result follows.

(b) Let $y \in Y$ and let $y=n_{0} a_{0} k_{0}$ be an Iwasawa decomposition for $y$. If $r \in R$ then $|\alpha|(y r)=|\alpha|(y)|| \alpha \mid\left(k_{0} r\right)$. Since $K R$ is a compact set, there exist positive constants $D_{1}<D_{2}$ such that $D_{1}<\left|\alpha\left(k_{0} r\right)\right|<D_{2}$ for all $k_{0} \in K$ and $r \in R$. By our assumption, there exists $r_{0} \in R$ such that $C_{1}<|\alpha|\left(y r_{0}\right)<C_{2}$. Hence $C_{1} / D_{2}<|\alpha|(y)<C_{2} / D_{1}$ and we can choose $C_{1}^{\prime}=C_{1} / D_{2}$ and $C_{2}^{\prime}=C_{2} / D_{1}$.

\section{Corollary 4.5.}

(a) The set $\mathcal{W}^{0}$ is invariant under right translations by $B$, i.e., if $W \in \mathcal{W}^{0}$ then for every $b \in B, W_{b} \in \mathcal{W}^{0}$ where $W_{b}(g)=W(g b)$.

(b) $W^{0}$ is invariant under right integration by compact open subset of closed subgroups of $B$, i.e., if $H$ is a closed subgroup of $B$ and $X \subset H$ is open and compact in $H$ then for every $W \in \mathcal{W}^{0}, W_{X} \in \mathcal{W}^{0}$ where $W_{X}(g)=\int_{X} W(g h) d h$.

Proof. (a) We take $R$ to be the Singleton, $R=\left\{b^{-1}\right\}$, where $b \in B$. By Lemma 4.4, $X_{C_{1}, C_{2}}(\alpha) b^{-1} \subset X_{C_{1}^{\prime}, C_{2}^{\prime}}(\alpha)$. Thus if $W$ restricted to the set $B w B$ is supported on $X_{C_{1}, C_{2}}(\alpha) \cap B w B$ then $W_{b}$ restricted to $B w B$ will be supported on the set $X_{C_{1}^{\prime}, C_{2}^{\prime}}(\alpha) \cap B w B$.

(b) Since $W$ is smooth on the right, $W_{X}$ is a finite linear combination of $W_{b_{i}}$ for some $b_{i} \in B$, hence (b) follows from (a). 
For each $w \in W$ we let $\mathcal{B}\left(S^{0}(w)\right)$ be the basis of $|V|$ defined in (2.10) and let $\mathcal{B}^{*}\left(S^{0}(w)\right)$ be the dual basis (up to scalars) that we fixed in (2.11). Let $M$ be a positive constant. We define the multiplicative cone $A^{M}(w) \subset A$ to be

$$
A^{M}(w)=\left\{a \in A:|\beta|(a)<M, \text { for all } \beta \in \mathcal{B}^{*}\left(S^{0}(w)\right)\right\} .
$$

Our first main theorem of this paper is the following:

Theorem 4.6. Let $W \in \mathcal{W}^{0}$ and $M$ a positive constant. Then the function

$$
(a, n) \mapsto W(a w n)
$$

defined on the set $A^{M}(w) \times N_{w}^{-}$is compactly supported in $N_{w}^{-}$. That is, if $W(a w n) \neq 0$ and $a \in A^{M}(w), n \in N_{w}^{-}$then $n$ is in some compact set independent of $a$.

Note that if $w=w_{0}$ then $S^{0}(w)=\emptyset$ hence $\mathcal{B}\left(S^{0}(w)\right)=\Delta^{*}$ and $\mathcal{B}^{*}\left(S^{0}(w)\right)$ $=\Delta$. It follows that $A^{M}(w)=A^{M}$ as defined in (1.2). Since $N_{w}^{-}=N$ in that case, Theorem 1.1 follows from the above theorem.

Proof. We prove the theorem for $w=e, w=w_{(1,2)}, w=w_{(1,2,3)}$ and $w=w_{(1,3)}=w_{0}$. The case $w=w_{(2,3)}$ is similar to the case $w=w_{(1,2)}$ and the case $w=w_{(1,3,2)}$ is similar to the case $w=w_{(1,2,3)}$, hence they will be omitted.

Case 1. $w=e$.

In this case, $N_{w}^{-}=\{e\}$ and there is nothing to prove.

Case 2. $w=w_{(1,2)}$.

In this case

$$
w=\left(\begin{array}{lll}
0 & 1 & 0 \\
1 & 0 & 0 \\
0 & 0 & 1
\end{array}\right)
$$

and $N_{w}^{-}=N_{1,2}$. We need to prove that $W\left(a w x_{1,2}(b)\right) \neq 0$ implies that there is a constant $C$ independent of $a \in A^{M}(w)$ such that $|b|<C$. Since $W$ is smooth on the right, there exist a constant $C_{1}$ such that $W\left(g x_{2,1}\left(-b^{-1}\right)\right)=$ $W(g)$ for all $|b|>C_{1}$ and all $g \in G$. Hence, if $|b|>C_{1}$ it follows from (2.6) that

$$
W\left(a w x_{1,2}(b) x_{2,1}\left(-b^{-1}\right)\right)=W\left(\widetilde{n} a h_{2,1}(b)\right)=\psi(\widetilde{n}) W\left(a h_{2,1}(b)\right)
$$

for some $\widetilde{n} \in N$. Hence $W\left(a w x_{1,2}(b)\right) \neq 0$ implies that $W\left(a h_{2,1}(b)\right) \neq 0$. We have that $S^{0}(e)=\Delta=\left\{|\alpha|_{1,2},|\alpha|_{2,3}\right\}$. Since $W\left(a h_{1,2}(b)\right) \neq 0$ and since $W \in \mathcal{W}^{0}$ it follows that both $|\alpha|_{1,2}$ and $|\alpha|_{2,3}$ are bounded on $a h_{2,1}(b)$. Hence $|\alpha|_{1,3}=|\alpha|_{1,2}+|\alpha|_{2,3}$ is bounded on $a h_{2,1}(b)$. Write $a=d\left(a_{1}, a_{2}, a_{3}\right)$. Then $|\alpha|_{1,3}\left(a h_{2,1}(b)\right)=\frac{\left|a_{1}\right|}{\left|b a_{3}\right|}$. Hence there exist positive constants $E_{1}$ and $E_{2}$ such 
that

$$
E_{1}<\frac{\left|a_{1}\right|}{\left|b a_{3}\right|}<E_{2}
$$

On the other hand $S^{0}\left(w_{1,2}\right)=\Delta$ and $B^{*}\left(S^{0}\left(w_{1,2}\right)\right)=\Delta^{*}=\left\{\lambda_{1}, \lambda_{2}\right\}$ (see (2.11)). Since $a \in A^{M}(w)$, we have that $\lambda_{1}(a)<M$ and $\lambda_{2}(a)<M$. Since $|\alpha|_{1,3}=\frac{1}{3}\left(\lambda_{1}+\lambda_{2}\right)$ we have that $|\alpha|_{1,3}(a)=\frac{\left|a_{1}\right|}{\left|a_{3}\right|}<M^{2 / 3}$. Combining this with (4.4) we get that $|b|<E_{1} M^{2 / 3}$.

Case 3. $w=w_{2,3}$.

The proof is similar to Case 2 .

Case 4. $w=w_{(1,2,3)}$.

In this case

$$
w=\left(\begin{array}{lll}
0 & 0 & 1 \\
1 & 0 & 0 \\
0 & 1 & 0
\end{array}\right)
$$

and $N_{w}^{-}=N_{2,3} N_{1,3}$. We need to prove that $W\left(a w x_{1,3}\left(b_{1}\right) x_{2,3}\left(b_{2}\right)\right) \neq 0$ implies that there is a constant $C$ independent of $a \in A^{M}(w)$ such that $\left|b_{1}\right|<C$ and $\left|b_{2}\right|<C$.

We first prove a special case. Assume that $W\left(a w x_{1,3}\left(b_{1}\right)\right) \neq 0$ and $a \in$ $A^{M}(w)$. If $\left|b_{1}\right|$ is large enough then we have

$$
W\left(a w x_{1,3}\left(b_{1}\right)\right)=W\left(a w x_{1,3}\left(b_{1}\right) x_{3,1}\left(-b_{1}^{-1}\right)\right)=\psi(\widetilde{n}) W\left(a h_{2,1}\left(b_{1}\right) w_{(2,3)}\right)
$$

for some $\widetilde{n} \in N$. Since $S^{0}\left(w_{(2,3)}\right)=\Delta, w_{(2,3)} \in K$ and $W \in \mathcal{W}^{0}$ it follows that $|\alpha|_{1,2}$ and $|\alpha|_{2,3}$ are bounded on $a h_{2,1}\left(b_{1}\right)$. In particular, $|\alpha|_{1,3}\left(a h_{2,1}\left(b_{1}\right)\right)$ $=\left(|\alpha|_{1,2}+|\alpha|_{2,3}\right)\left(a h_{2,1}\left(b_{1}\right)\right)=\left|a_{1} a_{3}^{-1} b_{1}^{-1}\right|$ is bounded from below.

On the other hand, $S^{0}\left(w_{1,2,3}\right)=\left\{|\alpha|_{2,3}\right\}=\left\{|\alpha|_{0,1,-1}\right\}$ hence $\mathcal{B}\left(S^{0}\left(w_{(1,2,3)}\right)\right)$ $=\left\{|\alpha|_{2,-1,-1},|\alpha|_{0,1,-1}\right\}$ and $\mathcal{B}^{*}\left(S^{0}\left(w_{1,2,3}\right)\right)=\left\{|\alpha|_{2,-1,-1},|\alpha|_{0,1,-1}\right\}$ (see (2.11)). Since $a \in A^{M}(w)$ we have that $|\alpha|_{1,3}(a)=\left(\frac{1}{2}\left(|\alpha|_{2,-1,-1}+|\alpha|_{0,1,-1}\right)\right)(a)=$ $\left|a_{1} a_{3}^{-1}\right|<M$ hence it follows that $\left|b_{1}\right|$ is bounded from above which is what we need.

For the general case, assume $W\left(a w x_{1,3}\left(b_{1}\right) x_{2,3}\left(b_{2}\right)\right) \neq 0$ and $a \in A^{M}(w)$. If $\left|b_{2}\right|$ is large enough then we can write

$$
\begin{aligned}
W\left(a w x_{1,3}\left(b_{1}\right) x_{2,3}\left(b_{2}\right)\right) & =W\left(a w x_{1,3}\left(b_{1}\right) x_{2,3}\left(b_{2}\right) x_{3,2}\left(-b^{-1}\right)\right) \\
& =\psi(\widetilde{n}) W\left(a h_{3,1}\left(b_{2}\right) w_{(1,2)} x_{1,2}\left(-b_{1} b_{2}^{-1}\right)\right)
\end{aligned}
$$

for some $\widetilde{n} \in N$. This lands us in Case 2! To use Case 2, we need to show that if $a \in A_{M}\left(w_{(1,2,3)}\right)$ and $\left|b_{2}\right|$ is large then $a h_{3,1}\left(b_{2}\right) \in A^{M_{1}}\left(w_{(1,2)}\right)$ for some positive constant $M_{1}$. Assume $\left|b_{2}\right|>D$. Then

$$
\begin{aligned}
|\alpha|_{2,-1,-1}\left(a h_{3,1}\left(b_{2}\right)\right) & =|\alpha|_{2,-1,-1}(a)|\alpha|_{2,-1,-1}\left(h_{3,1}\left(b_{2}\right)\right) \\
& =|\alpha|_{2,-1,-1}(a)|| b_{2}^{-3} \mid<M D^{-3} .
\end{aligned}
$$


Also

$$
\begin{aligned}
|\alpha|_{1,1,-2}\left(a h_{3,1}\left(b_{2}\right)\right) & =|\alpha|_{1,1,-2}(a)|\alpha|_{1,1,-2}\left(h_{3,1}\left(b_{2}\right)\right) \\
& =\left(\frac{1}{2}\left(|\alpha|_{2,-1,-1}+3|\alpha|_{0,1,-1}\right)\right)(a)\left|b_{2}^{-3}\right|<M^{2} D^{-3} .
\end{aligned}
$$

Hence we can choose $M_{1}=\max \left\{M D^{-3}, M^{2} D^{-3}\right\}$.

It follows that we can now use Case 2 to conclude that

$$
W\left(a h_{3,1}\left(b_{2}\right) w_{(1,2)} x_{1,2}\left(-b_{1} b_{2}^{-1}\right)\right) \neq 0
$$

with the above conditions on $a$ and $b_{2}$ implies that $\left|-b_{1} b_{2}^{-1}\right|$ is bounded.

Now $S^{0}\left(w_{1,2}\right)=\Delta$. Since $W \in \mathcal{W}^{0}$ it follows that $|\alpha|_{2,3}$ is bounded on $a h_{3,1}\left(b_{2}\right) w_{(1,2)} x_{1,2}\left(-b_{1} b_{2}^{-1}\right)$. (Here $b_{1}$ and $b_{2}$ and $a$ are allowed to change but are subject to the conditions $\left|b_{2}\right|>C, a \in A^{M}(w)$ and $W\left(a h_{3,1}\left(b_{2}\right) w_{(1,2)} x_{1,2}\left(-b_{1} b_{2}^{-1}\right)\right) \neq 0$. $)$ Since $\left|-b_{1} b_{2}^{-1}\right|$ is bounded it follows from Lemma 4.4 (b) that $|\alpha|_{2,3}$ is bounded on $a h_{3,1}\left(b_{2}\right)$. In particular, $|\alpha|_{2,3}\left(a h_{3,1}\left(b_{2}\right)\right)=\left|a_{2} a_{3}^{-1} b_{2}^{-1}\right|$ is bounded from below. Since $a \in A^{M}(w)$ we get that $|\alpha|_{2,3}(a)=\left|a_{2} a_{3}^{-1}\right|<M$ hence we get that $\left|b_{2}\right|$ is bounded from above (independent of $b_{1}$ or $a \in A^{M}(w)$ ).

So we can assume that $\left|b_{2}\right| \leq C_{1}$ for some positive constant $C_{1}$. Since $W$ is smooth on the right it follows that the space spanned by the functions $\left\{\rho\left(x_{2,3}\left(b_{2}\right)\right) W:\left|b_{2}\right| \leq C_{1}\right\}$ is finite dimensional. Here $(\rho(h) W)(g)=$ $W(g h)$. Let $W_{1}, \ldots, W_{l}$ be a basis for this space. It follows from Corollary 4.5 (a) that $W_{i}, i=1, \ldots, l$ are all in $\mathcal{W}^{0}$. By the special case above it follows that if $a \in A^{M}(w)$ and $W_{i}\left(a w x_{1,3}\left(b_{1}\right)\right) \neq 0$ the there exist a constant $D_{i}$ independent of $a$ such that $\left|b_{1}\right|<D_{i}$. Let $D$ be the maximum of the $D_{i}$ s. We write

$$
\begin{aligned}
W\left(a w x_{1,3}\left(b_{1}\right) x_{2,3}\left(b_{2}\right)\right) & =\left(\rho\left(x_{2,3}\left(b_{2}\right)\right) W\right)\left(a w x_{1,3}\left(b_{1}\right)\right) \\
& =\sum_{i} \lambda_{i} W_{i}\left(a w x_{1,3}\left(b_{1}\right)\right)
\end{aligned}
$$

where $\lambda_{i}$ is a scalar depending on $b_{2}$. Since $W\left(a w x_{1,3}\left(b_{1}\right) x_{2,3}\left(b_{2}\right)\right) \neq 0$, it follows that there exist $i$ such that $W_{i}\left(a w x_{1,3}\left(b_{1}\right)\right) \neq 0$ hence $\left|b_{1}\right|<D$. We notice that $D$ is independent of $\left|b_{2}\right| \leq C_{1}$.

Case 5. $w=w_{(1,3,2)}$.

This case is proved in the same way as Case 4 .

Case 6. $w=w_{(1,3)}=w_{0}$.

This case is similar to Case 4 . We will outline the proof:

In this case $N_{w}^{-}=N$. We need to prove that

$$
W\left(a w x_{1,3}\left(b_{1}\right) x_{2,3}\left(b_{2}\right) x_{1,2}\left(b_{3}\right)\right) \neq 0
$$

implies that there is a constant $C$ independent of $a \in A^{M}(w)$ such that $\left|b_{1}\right|<C,\left|b_{2}\right|<C$ and $\left|b_{3}\right|<C$. We first prove two special cases. In the 
first case we show that $W\left(a w x_{1,3}\left(b_{1}\right)\right) \neq 0$ implies that there is a constant $C$ independent of $a \in A^{M}(w)$ such that $\left|b_{1}\right|<C$. In the second case we show that $W\left(a w x_{1,3}\left(b_{1}\right) x_{2,3}\left(b_{2}\right)\right) \neq 0$ implies that there is a constant $C$ independent of $a \in A^{M}(w)$ such that $\left|b_{1}\right|<C$ and $\left|b_{2}\right|<C$. The proof follows the same lines as in Case 4 and is omitted.

For the general case we assume that $\left|b_{3}\right|$ is large. Then

$$
a w x_{1,3}\left(b_{1}\right) x_{2,3}\left(b_{2}\right) x_{1,2}\left(b_{3}\right) x_{2,1}\left(-b_{3}^{-1}\right)=n^{\prime} a h_{3,2}\left(b_{3}\right) w_{(1,2,3)} n^{\prime \prime}
$$

for some $n^{\prime} \in N$ and $n^{\prime \prime} \in N_{w_{(1,2,3)}}^{-}$. Hence

$$
\begin{aligned}
& W\left(a w x_{1,3}\left(b_{1}\right) x_{2,3}\left(b_{2}\right) x_{1,2}\left(b_{3}\right)\right) \\
& =W\left(a w x_{1,3}\left(b_{1}\right) x_{2,3}\left(b_{2}\right) x_{1,2}\left(b_{3}\right) x_{2,1}\left(-b_{3}^{-1}\right)\right) \\
& =\psi\left(n^{\prime}\right) W\left(a h_{3,2}\left(b_{3}\right) w_{(1,3,2)} n^{\prime \prime}\right) .
\end{aligned}
$$

Here we landed in Case 4. The proof continues as in the general step of Case 4.

\section{Projection into $\mathcal{W}^{0}(G, \psi)$.}

In this section we shall show that every $W \in \mathcal{W}(G, \psi)$ can be projected into $\mathcal{W}^{0}(G, \psi)$ by integrating it on a compact unipotent group versus a character of that group. We start with some preliminary results about Howe vectors.

5.1. Howe vectors. For a positive integer $m$ we denote by $K_{m}$ the congruence subgroup of $K$ given by $K_{m}=I_{3}+M_{3}\left(P^{m}\right)$. We let $A_{m}=A \cap K_{m}$. Let

$$
d=\left(\begin{array}{ccc}
1 & & \\
& \varpi^{2} & \\
& & \varpi^{4}
\end{array}\right) .
$$

Let $J_{m}=d^{m} K_{m} d^{-m}$. Then $J_{m}$ is given by

$$
J_{m}=I_{3}+\left(\begin{array}{ccc}
P^{m} & P^{-m} & P^{-3 m} \\
P^{3 m} & P^{m} & P^{-m} \\
P^{5 m} & P^{3 m} & P^{m}
\end{array}\right) .
$$

Notice that $J_{m}$ is expanding above the main diagonal and shrinking on and below the main diagonal. Let

$$
N_{m}=N \cap J_{m}
$$

Let $\bar{N}_{m}=\bar{N} \cap J_{m}$ and $\bar{B}_{m}=\bar{B} \cap J_{m}$. It is easy to see that

$$
J_{m}=\bar{N}_{m} A_{m} N_{m}=\bar{B}_{m} N_{m} \text {. }
$$

We fix a character $\psi_{k}$ on $k$ as Section 4. In particular $\psi_{k}(O)=1$ and $\psi_{k}\left(P^{-1}\right) \neq 1$. Let $\psi$ be a character of $N$ obtained from $\psi$ as in (4.1). For $m \geq 1$ we define a character $\psi_{m}$ on $J_{m}$ by

$$
\psi_{m}(j)=\psi\left(n_{j}\right)
$$


where $j=\bar{b}_{j} n_{j}, \bar{b}_{j} \in \bar{B}_{m}, n_{j} \in N_{m}$ is the unique decomposition of $j$. It is easy to see that $\psi_{m}$ is a character on $J_{m}$. For each $W \in \mathcal{W}(G, \psi)$ we define $W_{m}=W_{N_{m}, \psi}$ by

$$
W_{m}(g)=W_{N_{m}, \psi}(g)=\int_{N_{m}} W(g n) \psi^{-1}(n) d n .
$$

Since $N_{m+1} \supset N_{m}$ it is a simple application of Fubini to show that if $m \geq k$ then

$$
W_{m}(g)=\operatorname{vol}\left(N_{k}\right)^{-1} \int_{N_{m}} W_{k}(g n) \psi^{-1}(n) d n .
$$

For $g_{1} \in G$ we let $\left(\rho\left(g_{1}\right) W\right)(g)=W\left(g g_{1}\right)$.

Lemma 5.1. Let $M$ be such that $\rho\left(K_{M}\right) W=W$ and let $m$ be an integer such that $m>3 M$. Then

$$
\rho(j) W_{m}=\psi_{m}(j) W_{m}, \quad j \in J_{m} .
$$

Proof. Define

$$
\widetilde{W}_{m}=\operatorname{vol}\left(\bar{B}_{m}\right)^{-1} \int_{J_{m}} \psi_{m}^{-1}(j) \rho(j) W_{m} d j
$$

It is clear that $\widetilde{W}_{m}$ satisfies (5.5). On the other hand we have

$$
\begin{aligned}
& \operatorname{vol}\left(\bar{B}_{m}\right)^{-1} \int_{J_{m}} \psi_{m}^{-1}(j) \rho(j) W_{m} d j \\
& =\operatorname{vol}\left(\bar{B}_{m}\right)^{-1} \int_{N_{m}} \int_{\bar{B}_{m}} \psi^{-1}(n) \rho(n) \rho(\bar{b}) W d n d b .
\end{aligned}
$$

Looking at (5.1) it is easy to see that $\bar{B}_{m} \subseteq K_{M}$, hence $\bar{b} \in \bar{B}_{m}$ fixes $W$, and we get that $\widetilde{W}_{m}=W_{m}$.

Formulating Lemma 5.1 for functions we get that

$$
W_{m}(g j)=\psi_{m}(j) W_{m}(g) \text { for all } g \in G, j \in J_{m} .
$$

We call a vector $W$ in a representation space of $G$ satisfying (5.5) (or (5.6)) a Howe vector. The above Lemma shows that if the representation space affords a nontrivial Whittaker functional then nonzero Howe vectors exist. This property and some uniqueness properties of Howe vectors for irreducible admissible representations of $\mathrm{GL}_{n}(k)$ were established in $[\mathbf{H}]$. We now continue to study the behavior of Whittaker functions satisfying (5.6).

Lemma 5.2. Let $m>0$ and assume that $W \in \mathcal{W}(G, \psi)$ satisfies (5.6). Let $a \in A$. Then $W(a) \neq 0$ implies that $\alpha_{1,2}(a) \in 1+P^{m}$ and $\alpha_{2,3}(a) \in 1+P^{m}$. In particular, $|\alpha|_{1,2}$ and $|\alpha|_{2,3}$ are bounded on a. 
Proof. Assume $|b| \leq q^{m}$. Then

$$
\psi(b) W(a)=W\left(a x_{1,2}(b)\right)=W\left(x_{1,2}\left(\alpha_{1,2}(a) b\right) a\right)=\psi\left(\alpha_{1,2}(a) b\right) W(a) .
$$

Since $W(a) \neq 0$ then $\psi(b)=\psi\left(\alpha_{1,2}(a) b\right)$ for every $b$ such that $|b| \leq q^{m}$ hence using that $\psi$ has conductor $O$ we get that $\alpha_{1,2}(a) \in 1+P^{m}$. A similar proof works for $\alpha_{2,3}$.

Let $\chi_{X}$ be the characteristic function of the set $X$.

Corollary 5.3. Assume that $W \in \mathcal{W}(G, \psi)$ satisfies (5.6). Then

$$
W\left(\begin{array}{ccc}
a_{1} & & \\
& a_{2} & \\
& & 1
\end{array}\right)=\chi_{1+P^{m}}\left(a_{1}\right) \chi_{1+P^{m}}\left(a_{2}\right) .
$$

Let $N_{2} \subset \mathrm{GL}(2, k)$ be the subgroup of upper unipotent matrices and let

$$
\bar{B}_{2, m}=\left\{\left(\begin{array}{cc}
x_{1} & 0 \\
x_{2} & x_{3}
\end{array}\right): x_{1}, x_{3} \in 1+P^{m}, x_{2} \in P^{3 m}\right\} .
$$

The following Lemma is not needed for the proof of the main results in this paper. It is needed for our result on Bessel distributions in [Ba2].

Lemma 5.4. Assume that $W \in \mathcal{W}(G, \psi)$ satisfies (5.6). Let $h \in \mathrm{GL}(2, k)$. Then

$$
W\left(\begin{array}{ll}
h & \\
& 1
\end{array}\right)=0
$$

if $h \notin N_{2} \bar{B}_{2, m}$. If $h=n \bar{b}$ for $n \in N_{2}$ and $\bar{b} \in \bar{B}_{2, m}$ then

$$
W\left(\begin{array}{cc}
h & \\
& 1
\end{array}\right)=\psi(n) W(e)
$$

where $\psi$ is the natural restriction of $\psi$ to $\mathrm{N}_{2}$.

Proof. Let

$$
h=\left(\begin{array}{ll}
h_{1,1} & h_{1,2} \\
h_{2,1} & h_{2,2}
\end{array}\right)
$$

and let

$$
\left(\begin{array}{ll}
I & y \\
& 1
\end{array}\right) \in J_{m}
$$

where

$$
y=\left(\begin{array}{l}
y_{1} \\
y_{2}
\end{array}\right)
$$


and the above matrix being in $J_{m}$ is equivalent to $y_{1} \in P^{-3 m}$ and $y_{2} \in P^{-m}$. We have

$$
\begin{aligned}
\psi\left(y_{2}\right) W\left(\begin{array}{ll}
h & \\
& 1
\end{array}\right) & =W\left(\left(\begin{array}{ll}
h & \\
& 1
\end{array}\right)\left(\begin{array}{ll}
I & y \\
& 1
\end{array}\right)\right) \\
& =W\left(\left(\begin{array}{cc}
I & h y \\
& 1
\end{array}\right)\left(\begin{array}{ll}
h & \\
& 1
\end{array}\right)\right) \\
& =\psi\left(h_{2,1} y_{1}+h_{2,2} y_{2}\right) W\left(\begin{array}{ll}
h & \\
& 1
\end{array}\right) .
\end{aligned}
$$

Assume that

$$
W\left(\begin{array}{cc}
h & \\
& 1
\end{array}\right) \neq 0 .
$$

Then we have $\psi\left(h_{2,1} y_{1}+\left(h_{2,2}-1\right) y_{2}\right)=1$ for all $y_{1}$ and $y_{2}$ as above hence by the assumptions on the conductor of $\psi$ we have that $h_{2,1} \in P^{3 m}$ and $h_{2,2} \in 1+P^{m}$. It follows that we can write

$$
h=\left(\begin{array}{cc}
y_{1} & y_{2} \\
0 & y_{3}
\end{array}\right)\left(\begin{array}{ll}
1 & 0 \\
x & 1
\end{array}\right)
$$

with $x \in P^{3 m}$. Using (5.6) and Corollary 5.3 we get that $h \in N_{2} \bar{B}_{2, m}$ hence we proved the first statement. Using the equivariance of $W$ on the left by $N$ and on the right by $J_{m}$ we get the second statement.

Lemma 5.5. Assume that $W \in \mathcal{W}(G, \psi)$ satisfies (5.6). Then $W\left(a w_{(1,2)}\right)$ $=0$ and $W\left(a w_{(2,3)}\right)=0$ for every $a \in A$.

Proof. Let $b \in P^{-m}$ be such that $\psi(b) \neq 1$. Then

$$
\begin{aligned}
\psi(b) W\left(a w_{(1,2)}\right) & =W\left(a w_{(1,2)} x_{2,3}(b)\right) \\
& =W\left(x_{1,3}\left(\alpha_{1,3}(a) b\right) a w_{(1,2)}\right)=W\left(a w_{(1,2)}\right) .
\end{aligned}
$$

Since $\psi(b) \neq 0$ we get that $W\left(a w_{(1,2)}\right)=0$. A similar proof will show that $W\left(a w_{2,3}\right)=0$.

Lemma 5.6. Assume that $W \in \mathcal{W}(G, \psi)$ satisfies (5.6). Then $W\left(a w_{(1,2,3)}\right)$ $\neq 0$ implies that $\alpha_{2,3}(a) \in 1+P^{m}$ and in particular $|\alpha|_{2,3}$ is bounded on a. Also, $W\left(\right.$ aw $\left._{(1,3,2)}\right) \neq 0$ implies that $\alpha_{1,2}(a) \in 1+P^{m}$ and in particular $|\alpha|_{1,2}$ is bounded on a.

Proof. Let $b \in P^{-m}$. Then

$$
\begin{aligned}
\psi(b) W\left(a w_{(1,2,3)}\right) & =W\left(a w_{(1,2,3)} x_{1,2}(b)\right) \\
& =W\left(x_{2,3}\left(\alpha_{2,3}(a) b\right) a w_{(1,2,3)}\right) \\
& =\psi\left(\alpha_{2,3}(a) b\right) W\left(a w_{(1,2,3)}\right) .
\end{aligned}
$$

Hence, $W\left(a w_{(1,2,3)}\right) \neq 0$ implies that $\psi(b)=\psi\left(\alpha_{2,3}(a) b\right)$ for all $b \in P^{-m}$ hence $\alpha_{2,3}(a) \in 1+P^{m}$. A similar proof works for the case where $W\left(a w_{(1,3,2)}\right)$ $\neq 0$. 
We now prove the second main theorem of this paper. It was stated in the introduction as Theorem 1.3. Let $\mathcal{W}^{0}(G, \psi)$ be the subspace of $\mathcal{W}(G, \psi)$ introduced in Definition 4.1.

Theorem 5.7. Let $W \in \mathcal{W}(G, \psi)$. Then there exist a positive integer $M$ such that $W_{m}=W_{N_{m}, \psi} \in \mathcal{W}^{0}(G, \psi)$ for every $m \geq M$.

Proof. We need to show that there exist $M$ such that for every fixed $m \geq M$ and every $w \in \mathbb{W}$, the support of $W_{m}$ in $B w B$ has bounded image under every $|\alpha| \in S^{0}(w)$. It is enough to show this for each individual cell and then take the maximum of these $M$ s that we obtain.

We now show that given $w \in \mathbb{W}$ there exist an integer $M$ such that if $m \geq M$ and if $W_{m}(g) \neq 0$ then $|\alpha|(g)$ is in a fixed bounded set in $\mathbf{R}^{*}$ for every $\alpha \in S^{0}(w)$.

Case 1. $w=e$.

In that case $S^{0}(w)=\left\{|\alpha|_{1,2},|\alpha|_{2,3}\right\}$. Let $M$ be such that $W_{m}$ is a Howe vector (that is satisfies (5.6)) for $m \geq M$. We need to show that if $g \in$ $B w B=B$ and $W_{m}(g) \neq 0$ then both $|\alpha|_{1,2}$ and $|\alpha|_{2,3}$ are bounded on $g$. Since $g \in B$ we can write $g=n a$ for $n \in N$ and $a \in A$. We have that $|\alpha|(g)=$ $|\alpha|(a)$ for every $|\alpha| \in|X|$. Also, $W_{m}(g)=\psi(n) W_{m}(a)$ hence $W_{m}(g) \neq 0$ implies that $W_{m}(a) \neq 0$. Now the theorem follows from Lemma 5.2.

Case 2. $w=w_{(1,2)}$.

In that case $S^{0}(w)=\left\{|\alpha|_{1,2},|\alpha|_{2,3}\right\}$. Let $M_{1}$ be such that $W_{m}$ is a Howe vector for $m \geq M_{1}$ and let $M=3 M_{1}$. Let $m \geq M$. Let $g \in B w B$ and assume $W_{m}(g) \neq 0$. We can write $g=\operatorname{naw}_{(1,2)} x_{1,2}(b)$ for some $n \in N$, $a \in A$ and $b \in k$. To make the proof more clear we shall consider three cases although two may suffice. The first case is when $|b| \geq q^{3 m}$. In that case $x_{2,1}\left(-b^{-1}\right) \in J_{m}$ hence by (5.6) we have

$$
W_{m}(g)=W\left(g x_{2,1}\left(-b^{-1}\right)\right)=W(\widetilde{g})
$$

where $\widetilde{g}=g x_{2,1}\left(-b^{-1}\right) \in B$. By Case 1 it follows that $W(\widetilde{g}) \neq 0$ implies that $|\alpha|_{1,2}(\widetilde{g})$ and $|\alpha|_{2,3}(\widetilde{g})$ are in a fixed compact set. Hence if we let $R=$ $\left\{x_{2,1}\left(-b^{-1}\right):|b| \geq q^{3 m}\right\} \cup\{e\}$ then $R$ is a compact set in $G$ and the above argument implies that for each $g$ of the above form such that $|b| \geq q^{3 m}$ and such that $W_{m}(g) \neq 0$ there exist $r=r_{g} \in R$ such that $|\alpha|_{1,2}(g r)$ and $|\alpha|_{2,3}(\mathrm{gr})$ are in fixed compact set of $\mathbf{R}^{*}$. Hence, by Lemma 4.4 (b) we get that $|\alpha|_{1,2}(g)$ and $|\alpha|_{2,3}(g)$ are in compact sets independent of $g$, (depending only on $m$ and $W$ ).

The second case is when $q^{m}<|b|<q^{3 m}$. By (5.4) we can write

$$
W_{m}(g)=\frac{1}{\operatorname{vol}\left(N_{M_{1}}\right)} \int_{N_{m}} W_{M_{1}}(g n) \psi^{-1}(n) d n .
$$

Hence, if $W_{m}(g) \neq 0$ there exist $n^{\prime} \in N_{m}$ such that $W_{M_{1}}\left(g n^{\prime}\right) \neq 0$. We have that $g n^{\prime}=n^{\prime \prime} a w_{(1,2)} x_{1,2}(\widetilde{b})$ for some $n^{\prime \prime} \in N$ and $\widetilde{b} \in k$ such that 
$|\widetilde{b}|=|b|$. Since $|b|>q^{m} \geq q^{M}=q^{3 M_{1}}$ it follows that $x_{2,1}\left(-\widetilde{b}^{-1}\right) \in J_{M_{1}}$ and we can use the argument above to conclude that $|\alpha|_{1,2}\left(g n^{\prime} x_{2,1}\left(-\widetilde{b}^{-1}\right)\right)$ and $|\alpha|_{2,3}\left(g n^{\prime} x_{2,1}\left(-\widetilde{b}^{-1}\right)\right)$ are in fixed compact sets in $\mathbf{R}^{*}$ independent of $g$. Let $Q=\left\{x_{2,1}(c):|c| \leq q^{-m}\right\}$. Then $R=N_{m} Q$ is a compact set in $G$ and we can use Lemma 4.4 (b) to conclude that $|\alpha|_{1,2}(g)$ and $|\alpha|_{2,3}(g)$ are in fixed compact sets in $\mathbf{R}^{*}$ for all such $g$.

The last case is when $|b| \leq q^{m}$. In that case $x_{1,2}(b) \in J_{m}$ and we have

$$
W_{m}(g)=W_{m}\left(\operatorname{naw}_{(1,2)} x_{1,2}(b)\right)=\psi(n) \psi_{k}(b) W_{m}\left(a w_{(1,2)}\right)=0
$$

where the last equality is Lemma 5.5.

Case 3. $w=w_{(2,3)}$.

This case is similar to Case 2 and is omitted.

Case $4 . \quad w=w_{(1,2,3)}$.

In this case $S^{0}(w)=\left\{|\alpha|_{2,3}\right\}$. Let $M_{2}$ be such that $W_{m}$ satisfies (5.6) and such that $W_{m}$ satisfies the support conditions for the theorem on the Bruhat cells covered by Cases 1, 2 and 3 for every $m \geq M_{2}$. (Since an appropriate $M$ exist for each case we can take $M_{2}$ to be the maximum of the three.) Let $M_{1}=3 M_{2}$ and $M=3 M_{1}=9 M_{2}$. We need to show that for a fixed $m \geq M,|\alpha|_{2,3}$ is bounded on the set of $g \in B w B$ such that $W_{m}(g) \neq 0$. We can write

$$
g=\operatorname{nawx}_{1,3}\left(b_{1}\right) x_{2,3}\left(b_{2}\right)
$$

where $n \in N, a \in A$ and $b_{1}, b_{2} \in k$. We first show that for a fixed $m \geq M_{1}$, $|\alpha|_{2,3}$ is bounded on the set of $g$ of the form (5.7) satisfying $W_{m}(g) \neq 0$ and $\left|b_{2}\right|>q^{m}$. In that case we can write

$$
W_{m}(g)=\frac{1}{\operatorname{vol}\left(N_{M_{2}}\right)} \int_{N_{m}} W_{M_{2}}(g n) \psi^{-1}(n) d n .
$$

Since $W_{m}(g) \neq 0$ we get that $W_{M_{2}}\left(g n^{\prime}\right) \neq 0$ for some $n^{\prime} \in N_{m}$. Writing $g n^{\prime}$ in the form (5.7) with $\widetilde{b}_{2}$ replacing $b_{2}$ it is easy to see that $\left|\widetilde{b}_{2}\right|=\left|b_{2}\right|>$ $q^{m} \geq q^{M_{1}}=q^{3 M_{2}}$. Hence $x_{3,2}\left(-\widetilde{b}_{2}^{-1}\right) \in J_{M_{2}}$. It follows that $W_{M_{2}}\left(g n^{\prime}\right)=$ $W_{M_{2}}\left(g n^{\prime} x_{3,2}\left(-\widetilde{b}_{2}^{-1}\right)\right) \neq 0$. However, $\widetilde{g}=g n^{\prime} x_{3,2}\left(-\widetilde{b}_{2}^{-1}\right) \in B w_{(1,2)} B$ and our assumptions on $M_{2}$ imply that $|\alpha|_{2,3}(\widetilde{g})$ is in a fixed compact set in $\mathbf{R}^{*}$ depending only on $M_{2}$. Hence by Lemma 4.4 (b) $|\alpha|_{2,3}(g)$ is in a fixed compact set depending only on $m$ and $M_{2}$.

Now assume $m \geq M, W_{m}(g) \neq 0$ and $g$ is of the form (5.7) with $\left|b_{2}\right| \leq q^{m}$. If $\left|b_{1}\right| \leq q^{3 m}$ then $x_{1,3}\left(b_{1}\right) x_{2,3}\left(b_{2}\right) \in J_{m}$ and we have

$$
W_{m}(g)=\psi(n) \psi_{k}\left(b_{1}\right) \psi_{k}\left(b_{2}\right) W\left(a w_{(1,2,3)}\right) \neq 0 .
$$

By Lemma 5.6 we have that $|\alpha|_{2,3}(a)$ is in a fixed compact set in $\mathbf{R}$. But $|\alpha|_{2,3}(a)=|\alpha|_{2,3}\left(g x_{2,3}\left(-b_{2}\right) x_{1,3}\left(-b_{1}\right)\right)=|\alpha|_{2,3}(g \widetilde{n})$ for $\widetilde{n} \in N_{m}$. Hence, by Lemma 4.4 (b) we get that $|\alpha|_{2,3}(g)$ is in a fixed compact set. 
Now assume $\left|b_{1}\right|>q^{3 m}$. As before we write

$$
W_{m}(g)=\frac{1}{\operatorname{vol}\left(N_{M_{1}}\right)} \int_{N_{m}} W_{M_{1}}(g n) \psi^{-1}(n) d n .
$$

Again we have that there exist $n^{\prime} \in N_{m}$ such that $W_{M_{1}}\left(g n^{\prime}\right) \neq 0$. Write

$$
g^{\prime}=g n^{\prime}=n^{\prime \prime} a w x_{1,3}\left(\widetilde{b}_{1}\right) x_{2,3}\left(\widetilde{b}_{2}\right)
$$

as in (5.7). It is easy to see that $\left|\widetilde{b}_{1}\right|=\left|b_{1}\right|>q^{3 m} \geq q^{3 M}=q^{9 M_{1}}$. We now separate into two cases. If $\left|\widetilde{b}_{2}\right|>q^{M_{1}}$ then we are in the case already discussed above hence we have that $|\alpha|_{2,3}\left(g n^{\prime}\right)$ is in a fixed compact set hence $|\alpha|_{2,3}(g)$ is in a fixed compact set. Now assume that $\left|\widetilde{b}_{2}\right| \leq q^{M_{1}}$. Then $x_{2,3}\left(\widetilde{b}_{2}\right) \in J_{m}$ and

$$
W_{M_{1}}\left(g^{\prime}\right)=\psi_{k}\left(\widetilde{b}_{2}\right) W_{M^{\prime}}\left(n^{\prime \prime} a w x_{1,3}\left(\widetilde{b}_{1}\right)\right) \neq 0 .
$$

Since $\left|\widetilde{b}_{1}\right| \geq q^{9 M_{1}}$ we have that $x_{3,1}\left(-\widetilde{b}_{1}^{-1}\right) \in J_{M_{1}}$. Hence

$$
W_{M_{1}}\left(n^{\prime \prime} a w x_{1,3}\left(\widetilde{b}_{1}\right)\right)=W_{M_{1}}\left(n^{\prime \prime} a w x_{1,3}\left(\widetilde{b}_{1}\right) x_{3,1}\left(-\widetilde{b}_{1}^{-1}\right)\right) \neq 0 .
$$

However, $g^{\prime \prime}=n^{\prime \prime} a w x_{1,3}\left(\widetilde{b}_{1}\right) x_{3,1}\left(-\widetilde{b}_{1}^{-1}\right) \in B w_{(2,3)} B$ and by our assumptions on $M_{1},|\alpha|_{2,3}\left(g^{\prime \prime}\right)$ is in a fixed compact set. Since $g^{\prime \prime}=g^{\prime} x_{3,1}\left(-\widetilde{b}_{1}^{-1}\right)=$ $g n^{\prime} x_{2,3}\left(-\widetilde{b}_{2}\right) x_{3,1}\left(-\widetilde{b}_{1}^{-1}\right)$ we get that $|\alpha|_{2,3}(g)$ is in a fixed compact set.

Case 5. $w=w_{(1,3,2)}$.

This case is similar to Case 4 and is omitted.

Case 6. $w=w_{(1,3)}=w_{0}$.

In this case, $S^{0}(w)=\emptyset$ and there is nothing to prove.

\section{Bessel functions.}

In this section we attach Bessel functions to generic representations of $G$. Each Bessel function will be defined on one of three Bruhat cells and will be left and right invariant by $(N, \psi)$. The most significant one is the Bessel function attached to the open Bruhat cell $B w_{0} B$.

Let $w \in \mathbb{W}$. Let $N_{w}^{+}$and $N_{w}^{-}$be the subgroups of $N$ as defined in (2.14). We define the subtorus $A_{w}$ to be

$$
A_{w}=\left\{a \in A: \psi(n)=\psi\left(n^{a w}\right), \text { for all } n \in N_{w}^{+}\right\} .
$$

Here $n^{g}=g n g^{-1}$. It is easy to see that $A_{e}=Z(G), A_{(1,2)}=A_{(2,3)}=\emptyset$, $A_{(1,2,3)}=\left\{d\left(a_{1}, a_{2}, a_{3}\right) \in A: a_{2}=a_{3}\right\}, A_{(1,3,2)}=\left\{d\left(a_{1}, a_{2}, a_{3}\right) \in A: a_{1}=\right.$ $\left.a_{2}\right\}$ and $A_{(1,3)}=A$.

Let $w=w_{(1,2,3)}$ or $w=w_{(1,3,2)}$. It is easy to check that $N_{w}^{+}$normalizes $N_{w}^{-}$. If $n^{+} \in N_{w}^{+}$then the mapping $u \mapsto n^{+} u\left(n^{+}\right)^{-1}$ is measure preserving 
on $N_{w}^{-}$and we also have

$$
\psi\left(n^{+} u\left(n^{+}\right)^{-1}\right)=\psi(u), \quad \text { for every } u \in N_{w}^{-} .
$$

Let $N_{m}$ be the open compact subgroup of $N$ defined in (5.2). Then $N_{m}=$ $\left(N_{m} \cap N_{w}^{+}\right)\left(N_{m} \cap N_{w}^{-}\right)$. We let $N_{m}^{+}=N_{m, w}^{+}=\left(N_{m} \cap N_{w}^{+}\right)$and $N_{m}^{-}=N_{m, w}^{-}=$ $\left(N_{m} \cap N_{w}^{-}\right)$. We let $d n=d n^{+} d n^{-}$be a Haar measure on $N$ where $d n^{+}$and $d n^{-}$are Haar measures on $N_{w}^{+}$and $N_{w}^{-}$respectively.

Proposition 6.1. Let $w \in \mathbb{W}$ and $g \in N A_{w} w N_{w}^{-}$. Let $W \in \mathcal{W}^{0}(G, \psi)$ and let $W_{m}=W_{N_{m}, \psi}$ be the function defined in (5.3). Then

$$
\int_{N_{w}^{-}} W(g n) \psi^{-1} n d n=\frac{1}{\operatorname{vol}\left(N_{m}\right)} \int_{N_{w}^{-}} W_{m}(g n) \psi^{-1}(n) d n
$$

Proof. By Theorem 4.6 the integrals above converge absolutely. If $w=e$ then $N_{w}^{-}=\{e\}$ and there is nothing to prove. If $w=w_{(1,2)}$ or $w=w_{(2,3)}$ then $A_{w}=\emptyset$ and there is nothing to prove. If $w=w_{(1,3)}$ then $N_{w}^{-}=N$ and the proposition follows from a simple use of Fubini's theorem. So we assume $w=w_{(1,2,3)}$ or $w=w_{(1,3,2)}$. We first notice that if $g=n_{1} a w n_{2}$ where $n_{1} \in N$ and $n_{2} \in N_{w}^{-}$then

$$
\int_{N_{w}^{-}} W(g n) \psi^{-1}(n) d n=\psi\left(n_{1}\right) \psi\left(n_{2}\right) \int_{N_{w}^{-}} W(a w n) \psi^{-1}(n) d n
$$

and similarly for the second integral. Hence, using the assumption on $g$, it is enough to prove the equality for $g=a w$ with $a \in A_{w}$. In that case we have

$$
\begin{gathered}
\frac{1}{\operatorname{vol}\left(N_{m}\right)} \int_{N_{w}^{-}} W_{m}(\text { awu }) \psi^{-1}(u) d u \\
=\frac{1}{\operatorname{vol}\left(N_{m}^{+}\right)} \frac{1}{\operatorname{vol}\left(N_{m}^{-}\right)} \int_{N_{w}^{-}}\left(\int_{N_{m}^{-}} \int_{N_{m}^{+}} W\left(\text { awun }^{+} n^{-}\right)\right. \\
\left.\cdot \psi^{-1}(u) \psi^{-1}\left(n^{+} n^{-}\right) d n^{+} d n^{-}\right) d u .
\end{gathered}
$$

It follows from Theorem 4.6 that the function $u \mapsto W\left(a w u n^{+} n^{-}\right)$is supported on a compact set in $N_{w}^{-}$independent of $n^{+} \in N_{m}^{+}$and $n^{-} \in N_{m}^{-}$ hence the above integral converges absolutely and we can use Fubini and the change of variables $u \mapsto\left(n^{+}\right)^{-1} u n^{+}$and (6.2) to get that the above 
integral equals

$$
\begin{gathered}
\frac{1}{\operatorname{vol}\left(N_{m}^{+}\right)} \frac{1}{\operatorname{vol}\left(N_{m}^{-}\right)} \int_{N_{m}^{-}} \int_{N_{m}^{+}}\left(\int_{N_{w}^{-}} W\left(a w n^{+}\left(n^{+}\right)^{-1} u n^{+} n^{-}\right)\right. \\
\left.\cdot \psi^{-1}(u) \psi^{-1}\left(n^{+} n^{-}\right) d u\right) d n^{+} d n^{-} \\
=\frac{1}{\operatorname{vol}\left(N_{m}^{+}\right)} \frac{1}{\operatorname{vol}\left(N_{m}^{-}\right)} \int_{N_{m}^{-}} \int_{N_{m}^{+}}\left(\int_{N_{w}^{-}} W\left(a w n^{+} u n^{-}\right)\right. \\
\left.\cdot \psi^{-1}(u) \psi^{-1}\left(n^{+}\right) \psi^{-}\left(n^{-}\right) d u\right) d n^{+} d n^{-} .
\end{gathered}
$$

By (6.1) it follows that if $a \in A_{w}$ and $n^{+} \in N_{w}^{+}$then $W\left(a w n^{+} u n^{-}\right)=$ $\psi\left(n^{+}\right) W\left(\right.$ awun $\left.^{-}\right)$. Hence the last integral equals

$$
\begin{aligned}
& \frac{1}{\operatorname{vol}\left(N_{m}^{-}\right)} \int_{N_{m}^{-}}\left(\int_{N_{w}^{-}} W\left(\text { awun }^{-}\right) \psi^{-1}\left(\text { un }^{-}\right) d u\right) d n^{-} \\
& =\frac{1}{\operatorname{vol}\left(N_{m}^{-}\right)} \int_{N_{m}^{-}}\left(W(a w u) \psi^{-1}(u) d u\right) d n^{-} \\
& =\int_{N_{w}^{-}} W(a w u) \psi^{-1}(u) d u^{-} .
\end{aligned}
$$

Let $w \in \mathbb{W}$ and $g \in N A_{w} w N_{w}^{-}$. Let $W \in \mathcal{W}(G, \psi)$. By Theorem 5.7 there exist $M$ such that $W_{m} \in \mathcal{W}^{0}$ for every $m \geq M$. Let $m \geq M$. We define

$$
J_{g, w}(W)=\frac{1}{\operatorname{vol}\left(N_{m}\right)} \int_{N_{w}^{-}} W_{m}(g n) \psi^{-1}(n) d n .
$$

By applying Proposition 6.1 to $F=W_{M}$ and using that $F_{m}=\operatorname{vol}\left(N_{M}\right) W_{m}$ if $m \geq M$ we see that the above integral equals

$$
\frac{1}{\operatorname{vol}\left(N_{M}\right)} \int_{N_{w}^{-}} W_{M}(g n) \psi^{-1}(n) d n
$$

hence it is independent of $m$.

Remark 6.2. It follows from Proposition 6.1 that if $W \in \mathcal{W}^{0}$ then

$$
J_{g, w}(W)=\int_{N_{w}^{-}} W(g n) \psi^{-1}(n) d n .
$$

Remark 6.3. For $w=w_{0}=w_{(1,3)}$ we have that $N_{w}^{-}=N$. In that case we have that if $f$ is a compactly supported function on $N$ then $\int_{N} f(n) d n=$ 
$\lim _{m \rightarrow \infty} \int_{N_{m}} f(n) d n$. Hence

$$
\begin{aligned}
J_{g, w}(W) & =\frac{1}{\operatorname{vol}\left(N_{M}\right)} \int_{N} W_{M}(g n) \psi^{-1}(n) d n \\
& =\frac{1}{\operatorname{vol}\left(N_{M}\right)} \lim _{m \rightarrow \infty} \int_{N_{m}} W_{M}(g n) \psi^{-1}(n) d n \\
& =\lim _{m \rightarrow \infty} W_{m}(g) \\
& =\lim _{m \rightarrow \infty} \int_{N_{m}} W(g n) \psi^{-1}(n) d n
\end{aligned}
$$

which can be viewed as a principal valued $p$-adic integral. (See Definition 1.5.)

Lemma 6.4. Let $n \in N$. Then $J_{g, w}(\rho(n) W)=\psi(n) J_{g, w}(W)$. Here $(\rho(n) W)(g)=W(g n)$.

Proof. Let $F=\rho(n) W$. Pick $m$ large enough so that $F_{m} \in \mathcal{W}^{0}$ and such that $n \in N_{m}$. For such $m$ we have that $F_{m}=\psi(n) W_{m}$ and the result follows from the definition of $J_{g, w}$.

6.1. Bessel functions. We now return to the setting of Section 1.1. Let $\pi$ be an irreducible admissible representation of $G$ on a complex vector space $V$. Let $L$ be a nonzero $\psi$ Whittaker functional on $V$. Let $\mathcal{W}(\pi, \psi)$ be the space of functions

$$
W_{v}(g)=L(\pi(g) v)
$$

for all $v \in V$. It is easy to see that $W_{v} \in \mathcal{W}(G, \psi)$ and that the space $\mathcal{W}(\pi, \psi)=\left\{W_{v}: v \in V\right\}$ is a subspace of $\mathcal{W}(G, \psi)$ which is isomorphic to $(\pi, V)$. $G$ acts on $\mathcal{W}(G, \psi)$ by right translations (that is, by $\rho$ ). Let $w \in W$ and $g \in N A_{w} w N_{w}^{-}$. It follows from Lemma 6.4 that the restriction of the functional $J_{g, w}$ defined in (6.3) to $\mathcal{W}(\pi, \psi)$ is a Whittaker functional. From the uniqueness of the Whittaker functional it follows that there exist a scalar $j_{\pi, \psi, w}(g)$ such that

$$
J_{g, w}(W)=j_{\pi, \psi, w}(g) W(e) .
$$

If $w=w_{0}=w_{(1,3)}$ then we let $j_{\pi, \psi}(g)=j_{\pi, \psi, w_{0}}(g)$ and we call $j_{\pi, \psi}(g)$ the Bessel function of $\pi$.

Remark 6.5. The Bessel functions defined above are independent of the choice of initial Whittaker functional $L$. They do depend on the choice of Haar measure for $N$.

We let $\mathcal{W}^{0}(\pi, \psi)=\mathcal{W}(\pi, \psi) \cap \mathcal{W}^{0}(G, \psi)$.

Remark 6.6. Since $L$ is assumed to be nonzero there exist $W \in \mathcal{W}(\pi, \psi)$ such that $W(e) \neq 0$. Since $W_{m}(e)=\operatorname{vol}\left(N_{m}\right) W(e)$ it follows from Theorem 5.7 that there exist $W \in \mathcal{W}^{0}(\pi, \psi)$ such that $W(e) \neq 0$. Hence, 
$\mathcal{W}^{0}(\pi, \psi) \neq\{0\}$. (This is Corollary 1.4.) If we choose $W \in \mathcal{W}^{0}(\pi, \psi)$ such that $W(e)=1$ we get that $J_{g, w}(W)=j_{\pi, \psi, w}(g)$. By Remark 6.2 it follows that for such $W$ we have

$$
j_{\pi, \psi, w}(g)=\int_{N_{w}^{-}} W(g n) \psi^{-1}(n) d n
$$

for every $g \in N A_{w} w N_{w}^{-}$.

Remark 6.7. It follows from (6.5) that $j_{\pi, \psi, w}(g)$ satisfies

$$
j_{\pi, \psi, w}\left(n_{1} g n_{2}\right)=\psi\left(n_{1}\right) \psi\left(n_{2}\right) j_{\pi, \psi, w}(g), \quad \text { for all } n_{1} \in N, n_{2} \in N_{w}^{-} \text {. }
$$

Remark 6.8. If $\pi$ is supercuspidal then it follows from Remark 4.2 that $\mathcal{W}^{0}(\pi, \psi)=\mathcal{W}(\pi, \psi)$. Hence $j_{\pi, \psi, w}$ is defined by the equation

$$
\int_{N_{w}^{-}} W(g n) \psi^{-1}(n) d n=j_{\pi, \psi, w}(g) W(e)
$$

for all $W \in \mathcal{W}(G, \psi)$ and $g \in N A_{w} w N_{w}^{-}$.

Lemma 6.9. $j_{\pi, \psi, w}$ is locally constant on $N A_{w} w N_{w}^{-}$.

Proof. Using the invariance of $j_{\pi, \psi, w}$ given by (6.6) it is enough to show that $j_{\pi, \psi, w}$ is locally constant on $A_{w} w$. We write $j_{\pi, \psi, w}$ as an integral of a function $W \in \mathcal{W}^{0}(\pi, \psi)$ as in (6.5). By Theorem $4.6 n \mapsto W($ awn $)$ is compactly supported on $N_{w}^{-}$uniformly for $a \in A^{M}(w)$ for every positive constant $M$. Since the sets $A^{M}(w)$ are open sets of $A$ which cover $A$ the result is clear.

We finish this section by describing the Bessel functions associated to the contragredient representation. Let $\pi$ be an irreducible admissible representation of $G$ with a $\psi$ Whittaker functional $L$ as above. Let $\hat{\pi}$ be the representation contragredient to $\pi$. Let $\tau$ be the involution of $G$ defined by

$$
\tau(g)=w_{0}^{t} g^{-1} w_{0}
$$

For each $W \in \mathcal{W}(\pi, \psi)$ we let $W^{\tau}(g)=W(\tau(g))$. By [J-PS-S] the mapping $W \mapsto W^{\tau}$ is a bijection between $\mathcal{W}(\pi, \psi)$ and $\mathcal{W}\left(\hat{\pi}, \psi^{-1}\right)$.

Lemma 6.10. The mapping above induces a bijection between $\mathcal{W}^{0}(\pi, \psi)$ and $\mathcal{W}^{0}\left(\hat{\pi}, \psi^{-1}\right)$.

Proof. If $g \in G$ is written in the Iwasawa decomposition in the form $g=n a k$ where $n$ is upper triangular $a$ is diagonal and $k \in \mathrm{GL}_{3}(O)$ then

$$
\tau(g)=\left(w_{0}^{t} n^{-1} w_{0}\right)\left(w_{0} a^{-1} w_{0}\right)\left(w_{0}^{t} k^{-1} w_{0}\right)
$$

is an Iwasawa decomposition for $\tau(g)$. If $w \in W$ and $g \in B w B$ then $\tau(g) \in B w_{0} w w_{0} B$. It is easy to check that $\alpha_{1,2}(a)=\alpha_{2,3}\left(w_{0} a^{-1} w_{0}\right)$ and that $\alpha_{1,2} \in S^{0}(w)$ if and only if $\alpha_{2,3} \in S^{0}\left(w_{0} w w_{0}\right)$. Hence if $W \in \mathcal{W}^{0}(\pi, \psi)$ and $W^{\tau}(g) \neq 0$ we get that each $\alpha \in S^{0}\left(w_{0} w w_{0}\right)$ is bounded on $w_{0} a^{-1} w_{0}$ hence each $\alpha \in S^{0}(w)$ is bounded on $a$. 
Lemma 6.11. $j_{\hat{\pi}, \psi^{-1}, w}(g)=j_{\pi, \psi, \tau(w)}(\tau(g)), \quad g \in N A_{w} w N$.

Proof. Let $W \in \mathcal{W}^{0}(\pi, \psi)$ such that $W(e)=1$. (There exists such $W$ by Remark 6.6.) Then by Lemma $6.10, W^{\tau} \in \mathcal{W}^{0}\left(\hat{\pi}, \psi^{-1}\right)$ and $W^{\tau}(e)=1$. By (6.5) we have

$$
\int_{N_{w}^{-}} W^{\tau}(g n) \psi(n) d n=j_{\hat{\pi}, \psi^{-1}, w}(g) .
$$

On the other hand

$$
\begin{aligned}
\int_{N_{w}^{-}} W^{\tau}(g n) \psi(n) d n & =\int_{N_{w}^{-}} W(\tau(g n)) \psi(n) d n \\
& =\int_{N_{w}^{-}} W(\tau(g) \tau(n)) \psi(n) d n \\
& =\int_{N_{w_{0} w w_{0}}} W(\tau(g) n) \psi^{-1}(n) d n \\
& =j_{\pi, \psi, \tau(w)}(\tau(g)) .
\end{aligned}
$$

Corollary 6.12. $j_{\hat{\pi}, \psi^{-1}}(g)=j_{\pi, \psi}\left(g^{-1}\right), \quad g \in B w_{0} B$.

Proof. Recall that $j_{\pi, \psi}(g)=j_{\pi, \psi, w_{0}}(g)$. Since $\tau\left(w_{0}\right)=w_{0}$, it follows from Lemma 6.11 that $j_{\hat{\pi}, \psi^{-1}}(g)=j_{\pi, \psi}(\tau(g))$. It is clear that both the functions on the right-hand side and of the left-hand side of the equality we are trying to prove are equivariant from the left and right by $\left(N, \psi^{-1}\right)$. Hence it is enough to prove the equality for $g=a w_{0}$. If $g=a w_{0}$ then $\tau(g)=g^{-1}$ and we are done.

\section{Orbital integrals.}

In this section we show that the Bessel functions defined in Section 6 are given locally by orbital integrals. These integrals were studied in $[\mathbf{J}-\mathbf{Y} \mathbf{1}]$. We will do this in two steps. We will show that the Bessel function restricted to a small open set around an arbitrary element $g \in G$ is given by an integral of a Whittaker function which is compactly supported mod $N$. That is, if we restrict ourselves to this small neighborhood, we can replace a Whittaker function in the representation space with a different Whittaker function (not necessarily in the representation space) which is compactly supported mod $N$. Then we use the fact that each Whittaker function which is compactly supported mod $N$ comes from an integral of a function in $C_{c}^{\infty}(G)$. We will start from the second part.

Let $\omega$ be a character of $Z$ and let $\mathcal{W}_{\omega}(G, \psi) \subseteq \mathcal{W}(G, \psi)$ be the subspace of functions $W \in \mathcal{W}(G, \psi)$ satisfying

$$
W(g z)=\omega(z) W(g) \quad g \in G, z \in Z .
$$


Let $C_{c}^{\infty}(G)$ be the space of locally constant functions on $G$ with compact support. For each $f \in C_{c}^{\infty}(G)$ we let

$$
W_{f}(g)=W_{f}^{\psi}(g)=\int_{N} f(n g) \psi^{-1}(n) d n .
$$

It is clear that $W_{f} \in \mathcal{W}(G, \psi)$. We also define

$$
W_{f, \omega}(g)=\int_{N} f(n z g) \psi^{-1}(n) \omega^{-1}(z) d n d z, \quad f \in C_{c}^{\infty}(G) .
$$

It is clear that $W_{f, \omega} \in \mathcal{W}_{\omega}(G, \psi)$. The image of these maps is well-known. We thank H. Jacquet for providing us with the following proof which we include for the sake of completeness:

Lemma 7.1. Let $f \in C_{c}^{\infty}(G)$. Then $W_{f}$ is compactly supported $\bmod N$ and the map $f \mapsto W_{f}$ is a linear map onto the space of compactly supported functions $\bmod N$ in $\mathcal{W}(G, \psi)$.

Proof. The only nontrivial claim is that the map is onto. Assume $W \in$ $\mathcal{W}(G, \psi)$ is compactly supported $\bmod N$. We would like to show that $W=W_{f}$ for some $f \in C_{c}^{\infty}(G)$. Since $W$ is smooth there exist an open compact group $U$ such that $W(g u)=W(u)$ for all $g \in G$ and $u \in U$. Since $W$ is compactly supported $\bmod N$, it follows that it is supported on a finite number of double cosets of the form $N g U$. Hence, it is enough to prove the result for $W$ which is supported on one double coset $N g U$. For such $W$ we have that $W(n g u)=\psi(n) W(g)$ for all $n \in N$ and $u \in U$ and $W(x)=0$ if $x \notin N g U$. Assume $W(g) \neq 0$. Then $\psi(n)=1$ for all $n \in g U g^{-1} \cap N$. Let $F$ be the characteristic function of $g U g^{-1} \cap N$ and let $c=\int_{N} F(n) d n$. Define $f \in C_{c}^{\infty}(G)$ by $f(n g u)=c^{-1} W(g) F(n)$, and $f(x)=0$ if $x \notin N g U$. It is easy to check that $f$ is well-defined and that $W_{f}=W$.

The same proof works for our second projection:

Lemma 7.2. Let $f \in S(G)$. Then $W_{f, \omega}$ is compactly supported $\bmod N Z$ and the map $f \mapsto W_{f, \omega}$ is a linear map onto the space of compactly supported functions $\bmod N$ in $\mathcal{W}_{\omega}(G, \psi)$.

Let $|V|$ be the subspace of $|X|$ given by $|V|=\left\{|\alpha|_{r_{1}, r_{2}, r_{3}}: r_{1}+r_{2}+r_{3}=0\right\}$. (See (2.7).) Let $Q=\left\{\beta_{1}, \beta_{2}\right\}$ be a basis for $|V|$. Let $C_{1}<C_{2}$ be positive constants and define

$$
A_{Q}\left(C_{1}, C_{2}\right)=\left\{a \in A: C_{1}<\beta_{i}(a)<C_{2}, i=1,2\right\} .
$$

Lemma 7.3. A function $W$ on $G$ is compactly supported $\bmod N Z$ if and only if there exist constants $C_{1}, C_{2}$ such that $W$ is supported on $N A_{Q}\left(C_{1}\right.$, $\left.C_{2}\right) K$. 
Proof. We can write $A_{Q}\left(C_{1}, C_{2}\right)=Z A^{\prime}$ where

$$
A^{\prime}=\left\{d\left(a_{1}, a_{2}, 1\right) \in A_{Q}\left(C_{1}, C_{2}\right)\right\} .
$$

Since $Q$ is a basis it is clear that $A^{\prime}$ is compact. Hence if $W$ is supported on $N A_{Q}\left(C_{1}, C_{2}\right) K$ then it is compactly supported $\bmod N Z$. Now assume $W$ is compactly supported $\bmod N Z$. Then $W$ is supported on a set of the form $N Z R$ for some compact set $R$. Since the sets $N A_{Q}\left(C_{1}, C_{2}\right) K$ for different choices of $C_{1}$ and $C_{2}$ are open sets that cover $G$ we get that the sets of the form $N A_{Q}\left(C_{1}, C_{2}\right) K$ cover $R$. Since $R$ is compact there exist constants $C_{1}^{\prime}, C_{2}^{\prime}$ so that $R \subset N A_{Q}\left(C_{1}^{\prime}, C_{2}^{\prime}\right) K$. Hence $N Z R \subset N A_{Q}\left(C_{1}^{\prime}, C_{2}^{\prime}\right) K$.

For each $w \in \mathbb{W}$ we define the set $M(w) \subset \Delta^{*}$ as follows:

$$
M(w)=\left\{\alpha^{*} \mid \alpha \in \Delta, \alpha \notin S^{0}(w)\right\} .
$$

Then $M(e)=M\left(w_{(1,2)}\right)=M\left(w_{2,3}\right)=\emptyset, M\left(w_{1,2,3}\right)=\left\{\lambda_{2}\right\}, M\left(w_{1,3,2}\right)=$ $\left\{\lambda_{1}\right\}$ and $M\left(w_{(1,3)}\right)=\Delta^{*}$.

Remark 7.4. It is easily checked that the set $M(w) \cup S^{0}(w)$ is a basis for $|V|$.

Let $E$ be a positive constant. We let

$$
A_{M(w)}(E)=\{a \in A:|\lambda|(a)>E \text { for every } \lambda \in M(w)\} .
$$

Theorem 7.5. Let $W \in \mathcal{W}^{0}, w \in \mathbb{W}$ and $E>0$. There exist a function $F \in \mathcal{W}^{0}$ compactly supported $\bmod N Z$ such that

$$
F\left(n_{1} a w n_{2}\right)=W\left(n_{1} a w n_{2}\right)
$$

for all $a \in A_{M(w)}(E)$ and $n_{1}, n_{2} \in N$.

Remark 7.6. Let $C_{1}<C_{2}$ be positive constants and let $A_{C_{1}, C_{2}}=A_{\Delta}\left(C_{1}\right.$, $C_{2}$ ). By Lemma 7.3 we have that $F$ being compactly supported $\bmod N Z$ is equivalent to $F$ being supported on a set of the form $N A_{C_{1}, C_{2}} K$ for some $C_{1}, C_{2}$. Hence we can find $F \in \mathcal{W}^{0}$ compactly supported $\bmod N Z$ such that (7.3) holds if and only if we can find constants $C_{1}, C_{2}$ such that the function

$$
F(g)= \begin{cases}W(g), & \text { if } g \in N A_{C_{1}, C_{2}} K \\ 0, & \text { otherwise, }\end{cases}
$$

satisfies (7.3). Hence, we shall use (7.4) to define the desired $F$. Notice that if we define $F$ by (7.4) then $W(g)=0 \Rightarrow F(g)=0$ hence we only need to prove (7.3) for $g=n_{1} a w n_{2}$ such that $a \in A_{M(w)}(E)$ and $W(g) \neq 0$.

Proof. We proceed with a case by case analysis as in the proofs of Theorem 3.1, Theorem 4.6 and Theorem 5.7. 
Case 1. $w=e$.

In this case $S^{0}(w)=\Delta, M(w)=\emptyset$ and $N_{w}^{-}=\{e\}$. Since $W \in \mathcal{W}^{0}$ it follows that the support of $W$ on $B$ is contained in a set of the form $N A_{C_{1}, C_{2}}$. Define $F$ as in (7.4). Then $F$ satisfies the requirements of the theorem.

Case 2. $w=w_{(1,2)}$.

In this case $S^{0}(w)=\Delta$ and $M(w)=\emptyset$. Since $W \in \mathcal{W}^{0}$ it follows that the support of $W$ on $B w B$ is contained in $N A_{C_{1}, C_{2}} K$ for some constants $C_{1}, C_{2}$. Hence, if we define $F$ by (7.4) then $F=W$ on $B w B$. Thus $F$ will satisfy the requirements of the theorem.

Case 3. $w=w_{(2,3)}$.

This case is the same as Case 2.

Case 4 . $w=w_{(1,2,3)}$.

In this case $S^{0}(w)=\left\{|\alpha|_{2,3}=|\alpha|_{0,1,-1}\right\}, M(w)=\left\{|\alpha|_{2,-1,-1}\right\}$ and $N_{w}^{-}=$ $N_{2,3} N_{1,3}$.

We consider a special case. Assume that $W\left(a w x_{1,3}(b)\right) \neq 0$ and $|\alpha|_{2,-1,-1}(a)>E$ (that is, $\left.a \in A_{M(w)}(E)\right)$. If $|b|$ is large then $x_{3,1}\left(-b^{-1}\right)$ stabilizes $W$ and we have

$$
W\left(a w x_{1,3}(b)\right)=W\left(a w x_{1,3}(b) x_{3,1}\left(-b^{-1}\right)\right) .
$$

Now $a w x_{1,3}(b) x_{3,1}\left(-b^{-1}\right) \in B w_{(2,3)} B$ and by Case 3 we can choose $F$ as in (7.4) so that $F(g)=W(g)$ for every $g \in B w_{(2,3)} B$. Since $F$ is smooth $F$ satisfies an equation such as (7.5) for $|b|$ large enough hence we have that there exist a constant $D$ such that

$$
F\left(a w x_{1,3}(b)\right)=W\left(a w x_{1,3}(b)\right)
$$

for every $|b|>D$.

Now assume $|b| \leq D$. It follows from the proof of Lemma 4.4 (a) that there exist a constant $E_{1}$ such that $|\alpha|_{2,-1,-1}\left(a w x_{1,3}(b)\right)>E_{1}$ for every $|b| \leq D$ and every $a$ such that $|\alpha|_{2,-1,-1}(a)>E$. Since we assumed that $W\left(a w x_{1,3}(b)\right) \neq 0$ and since $|\alpha|_{2,-1,-1}$ is a positive linear combination of $|\alpha|_{1,2}$ and $\left|\alpha_{2,3}\right|$ it follows from [J-PS-S] (see also Remark 4.2) that there exist a constant $E_{2}$ such that $|\alpha|_{2,-1,-1}\left(a w x_{1,3}(b)\right)<E_{2}$. Since $W \in \mathcal{W}^{0}$ there exist constants $D_{1}, D_{2}$ such that $D_{1}<|\alpha|_{0,1,-1}\left(a w x_{1,3}(b)\right)<D_{2}$. Hence it follows from Lemma 7.3 that $a w x_{1,3}(b) \in N A_{C_{1}, C_{2}} K$ for some constants $C_{1}, C_{2}$ and if we define $F$ by (7.4) we get that

$$
F\left(a w x_{1,3}(b)\right)=W\left(a w x_{1,3}(b)\right)
$$

for every $a \in A$ such that $|\alpha|_{2,-1,-1}(a)>E$ and every $b \in k$ such that $|b| \leq D$. From (7.6), and (7.7) it follows that we can choose the constants $C_{1}$ and $C_{2}$ to cover both cases and then the $F$ defined by (7.4) will satisfy

$$
F\left(a w x_{1,3}(b)\right)=W\left(a w x_{1,3}(b)\right)
$$


for every $a \in A$ such that $|\alpha|_{2,-1,-1}(a)>E$ and every $b \in k$.

For the general case we consider the values of the function

$$
W\left(a w x_{1,3}\left(b_{1}\right) x_{2,3}\left(b_{2}\right)\right),
$$

with $|\alpha|_{2,-1,-1}(a)>E$. If $\left|b_{2}\right|$ is very large then we argue as in the special case that there exist constants $C_{1}, C_{2}$ and a function $F$ given by (7.4) such that

$$
F\left(a w x_{1,3}\left(b_{1}\right) x_{2,3}\left(b_{2}\right)\right)=W\left(a w x_{1,3}\left(b_{1}\right) x_{2,3}\left(b_{2}\right)\right)
$$

for every for every $a \in A$ such that $|\alpha|_{2,-1,-1}(a)>E$ and every $b_{2} \in k$ such that $\left|b_{2}\right|$ is larger than some constant $D$.

Assume $\left|b_{2}\right| \leq D$. There exist functions $W_{1}, \ldots, W_{l} \in \mathcal{W}^{0}$ such that

$$
\rho\left(x_{2,3}\left(b_{2}\right)\right) W=c_{1}(b) W_{1}+\cdots+c_{l}(b) W_{l}
$$

for all $\left|b_{2}\right| \leq D$ and $c_{i}$ are locally constant functions from $\{x:|x| \leq C\}$ to $\mathbb{C}$. By our special case, there exist functions $F_{1}, \ldots, F_{l} \in \mathcal{W}^{0}$ compactly supported $\bmod N Z$ such that

$$
F_{i}\left(a w x_{1,3}\left(b_{1}\right)\right)=W_{i}\left(a w x_{1,3}\left(b_{1}\right)\right)
$$

for every $a \in A$ such that $|\alpha|_{2,-1,-1}(a)>E$. Let $y \in k$ with $|y| \leq D$ and define

$$
F_{y}=c_{1}(y) \rho\left(x_{2,3}(-y)\right) F_{1}+\cdots+c_{l}(y) \rho\left(x_{2,3}(-y)\right) F_{l} .
$$

Then we have

$$
F_{y}\left(a w x_{1,3}\left(b_{1}\right) x_{2,3}(y)\right)=W\left(a w x_{1,3}\left(b_{1}\right) x_{2,3}(y)\right)
$$

for every $a \in A$ such that $|\alpha|_{2,-1,-1}(a)>E$. Since every function in sight is locally constant the equality in (7.10) will remain true if we fix $F_{y}$ but change $x_{2,3}(y)$ to $x_{2,3}\left(y^{\prime}\right)$ for some $y^{\prime}$ in a small neighborhood of $y$. Since $F_{y}$ is compactly supported mod $N Z$ it follows from Remark 7.6 that we can choose $F$ as in (7.4) so that (7.9) will hold for every $a$ as above and every $\left|b_{2}\right| \leq D$ in a small neighborhood of $y$. Since we need only a finite number of such $y$ to cover the set $\left\{b_{2}:\left|b_{2}\right| \leq D\right\}$ we can change the constants $C_{1}$ and $C_{2}$ in the definition of $F$ in (7.4) to get the conclusion of the theorem.

Case 5. $w=w_{(1,3,2)}$.

This case is similar to Case 4 and is omitted.

Case 6. $w=w_{0}=w_{(1,3)}$.

In this case $S^{0}(w)=\emptyset$ hence $M(w)=\Delta^{*}=\left\{\lambda_{1}, \lambda_{2}\right\}$. Also $N_{w}^{-}=N$. We first consider two special cases. Since the arguments are the same as in Case 4 and as in our general case below we omit them.

In the first special case we prove that there exist constants $C_{1}, C_{2}$ depending on $E$ such that if $F$ is given by (7.4) then

$$
F\left(a w x_{1,3}(b)\right)=W\left(a w x_{1,3}(b)\right)
$$


for every $a \in A$ such that $\lambda_{1}(a)>E$ and $\lambda_{2}(a)>E$. The proof of this case is the same as the proof of the special case of Case 4 .

In the second special case we prove that there exist constants $C_{1}, C_{2}$ depending on $E$ such that if $F$ is given by (7.4) then

$$
F\left(a w x_{1,3}\left(b_{1}\right) x_{2,3}\left(b_{2}\right)\right)=W\left(a w x_{1,3}\left(b_{1}\right) x_{2,3}\left(b_{2}\right)\right)
$$

for every $a \in A$ such that $\lambda_{1}(a)>E$ and $\lambda_{2}(a)>E$. The proof of this case uses the first special case and is similar to our general case below.

We consider the function $W\left(a w x_{1,3}\left(b_{1}\right) x_{2,3}\left(b_{2}\right) x_{1,2}\left(b_{3}\right)\right)$. If $\left|b_{3}\right|$ is very large then

$$
W\left(a w x_{1,3}\left(b_{1}\right) x_{2,3}\left(b_{2}\right) x_{1,2}\left(b_{3}\right)\right)=W\left(a w x_{1,3}\left(b_{1}\right) x_{2,3}\left(b_{2}\right) x_{1,2}\left(b_{3}\right) x_{2,1}\left(-b_{3}^{-1}\right)\right) .
$$

Let $g=a w x_{1,3}\left(b_{1}\right) x_{2,3}\left(b_{2}\right) x_{1,2}\left(b_{3}\right) x_{2,1}\left(-b_{3}^{-1}\right)$. Then we can write $g=$ $n_{1} \widetilde{a} w_{(1,2,3)} n_{2}$ with $n_{1} \in N, \widetilde{a} \in A$ and $n_{2} \in N_{w_{(1,2,3)}}^{-}$. It is also easy to see that $\widetilde{a}=a h_{3,2}\left(b_{2}\right)$. Since $\lambda_{1}\left(a h_{3,2}\left(b_{2}\right)\right)=\lambda_{1}(a)$ it follows from Case 4 that there exist constants $C_{1}, C_{2}$ depending on $E$ such that if $F$ is defined by (7.4) then

$$
F\left(n_{1} a h_{3,2}\left(b_{2}\right) w_{(1,2,3)} n_{2}\right)=W\left(n_{1} a h_{3,2}\left(b_{2}\right) w_{(1,2,3)} n_{2}\right)
$$

for every $|a|$ such that $\lambda_{1}(a)>E$. Since $F$ is smooth it follows that there exist a constant $|D|$ such that

$$
F\left(a w x_{1,3}\left(b_{1}\right) x_{2,3}\left(b_{2}\right) x_{1,2}\left(b_{3}\right)\right)=W\left(a w x_{1,3}\left(b_{1}\right) x_{2,3}\left(b_{2}\right) x_{1,2}\left(b_{3}\right)\right)
$$

for every $a \in A$ and $b_{3} \in k$ such that $\lambda_{1}(a)>E$ and $\left|b_{3}\right|>D$. For the case where $\left|b_{3}\right| \leq D$ we argue as in Case 4 using our special case above.

For $W \in \mathcal{W}^{0}$ and $g \in B w_{0} B$ we let

$$
J(W, g)=J_{g, w_{0}}(W)=\int_{N} W(g n) \psi^{-1}(n) d n .
$$

Corollary 7.7. Let $W \in \mathcal{W}^{0}$ and $x \in G$. Let $U_{x}$ be a compact neighborhood of $x$ in $G$. Then there exists a function $F \in \mathcal{W}$ compactly supported mod $N Z$ such that

$$
J(F, g)=J(W, g)
$$

for every $g \in U_{x} \cap B w_{0} B$.

Proof. Let $\Delta^{*}=\left\{\lambda_{1}, \lambda_{2}\right\}$ as above. Since $U_{x}$ is compact it follows that $\lambda_{1}$ and $\lambda_{2}$ are both bounded on $U_{x}$. Hence there exist a constant $E$ such that $\lambda_{1}(g)>E$ and $\lambda_{2}(g)>E$ for every $g \in U_{x}$. Let $g \in U_{x} \bigcap B w_{0} B$. Then $g=n_{1} a w_{0} n_{2}$ for some $n_{1}, n_{2} \in N$ and $a \in A$. By Theorem 3.1 we have

$$
\lambda_{i}(g)=\lambda_{i}\left(n_{1} a w_{0} n_{2}\right)=\lambda_{i}(a) \lambda\left(w_{0} n_{2}\right) \leq \lambda_{i}(a)
$$

for $i=1,2$. Since $\lambda_{i}(g)>E$ it follows that $\lambda_{i}(a)>E$ for $i=1,2$. By Theorem 7.5 we can find $F$ in $\mathcal{W}$ compactly supported $\bmod N Z$ such that

$$
F\left(a w_{0} n\right)=W\left(a w_{0} n\right)
$$


for every $n \in N$ and $a \in A$ such that $\lambda_{i}(a)>E, i=1,2$. In particular we have $F(g n)=W(g n)$ for every $g \in U_{x} \cap B w_{0} B$ and $n \in N$. Hence $J(F, g)=J(W, g)$ for such $g$ which is what we wanted to prove.

Let $\phi \in C_{c}^{\infty}(G)$ and $\omega$ a quasi-character of $Z$. Let $g \in B w_{0} B$. We define

$$
J_{\psi, \omega}(\phi, g)=\int_{N} \int_{N} \int_{Z} f\left(n_{1} z g n_{2}\right) \psi^{-1}\left(n_{1}\right) \psi^{-1}\left(n_{2}\right) \omega^{-1}(z) d n_{1} d n_{2} d z .
$$

It follows from $[\mathbf{J}-\mathbf{Y} \mathbf{1}]$ that this integral converges absolutely. It is easy to see that

$$
J_{\psi, \omega}(\phi, g)=\int_{N} W_{\phi, \omega}(g n) \psi^{-1}(n) d n=J\left(W_{\phi, \omega}, g\right)
$$

where $W_{\phi, \omega}$ is defined by (7.2).

Corollary 7.8. Let $\pi$ be an irreducible admissible representation of $G$ with central character $\omega_{\pi}$. Let $x \in G$ and let $U_{x}$ be a compact neighborhood of $x$ in $G$. Then there exist a function $\phi \in C_{c}^{\infty}(G)$ such that

$$
J_{\psi, \omega_{\pi}}(\phi, g)=j_{\pi, \psi}(g)
$$

for every $g \in U_{x} \cap B w_{0} B$.

Proof. Recall that $j_{\pi, \psi}(g)=j_{\pi, \psi, w_{0}}(g)$ is the Bessel function associated to the longest Weyl element $w_{0}$. By (6.5) there exist $W \in \mathcal{W}^{0}(\pi, \psi)$ such that $j_{\pi, \psi}(g)=J(W, g)$ for every $g \in B w_{0} B$. Since the central character of $\pi$ is $\omega_{\pi}$ it follows that $W \in \mathcal{W}_{\omega_{\pi}}^{0}(G, \psi)=\mathcal{W}_{\omega_{\pi}}(G, \psi) \cap \mathcal{W}^{0}(G, \psi)$. By Corollary 7.7 there exist $F \in \mathcal{W}(G, \psi)$ which is compactly supported $\bmod N Z$ such that $J(F, g)=J(W, g)$ for every $g \in U_{x} \cap B w_{0} B$. It is easy to see from the proof of Theorem 7.5 that if $W \in \mathcal{W}_{\omega}^{0}(G, \psi)$ then $F$ can be chosen in $\mathcal{W}_{\omega}(G, \psi)$ (see (7.4)). By Lemma 7.2 there exist $\phi \in C_{c}^{\infty}(G)$ such that $W_{\phi, \omega}=F$. Hence

$$
J_{\psi, \omega}(\phi, g)=J\left(W_{\phi, \omega}, g\right)=J(F, g)=J(W, g)=j_{\pi, \psi}(g)
$$

for every $g \in U_{x} \cap B w_{0} B$.

Remark 7.9. If $\pi$ is supercuspidal then there exis $\phi \in C_{c}^{\infty}(G)$ such that (7.12) holds for every $g \in B w_{0} B$. That is, in this case the Bessel function is globally given by an orbital integral. This follows from Remark 4.2, from Lemma 7.2 and (6.5).

Acknowledgments. I thank J. Cogdell, H. Jacquet, I. Piatetski-Shapiro, and S. Rallis for sharing their insight with me and their constant encouragement. 


\section{References}

[Av] Z. Averbuch, Bessel functions of algebraic groups' representations over a local field, Tel Aviv University, 1992.

[Ba] E.M. Baruch, On Bessel distributions for quasi-split groups, Trans. Amer. Math. Soc., 353(7) (2001), 2601-2614, MR 2002b:22029, Zbl 0976.22015.

[Ba2] , Bessel distributions for GL(3) over the p-adics, Pacific J. Math., to appear.

[Bo] A. Borel, Introduction aux Groupes Arithmétiques, Hermann, Paris, 1969, MR 39 \#5577, Zbl 0186.33202.

[Bo-HC] A. Borel and Harish-Chandra, Arithmetic subgroups of algebraic groups, Ann. of Math. (2), 75 (1962), 485-535, MR 26 \#5081, Zbl 0107.14804.

[C-PS1] J.W. Cogdell and I. Piatetski-Shapiro, The Arithmetic and Spectral Analysis of Poincaré Series, Perspectives in Mathematics, 13, Academic Press Inc., Boston, MA, 1990, MR 91h:11042, Zbl 0714.11032.

[G-PS] S. Gelbart and I. Piatetski-Shapiro, Distinguished representations and modular forms of half-integral weight, Invent. Math., 59(2) (1980), 145-188, MR 82b:10035, Zbl 0426.10027.

[HC] Harish-Chandra, Admissible Invariant Distributions on Reductive p-adic Groups, University Lecture Series, 16, American Mathematical Society, Providence, RI, 1999, MR 2001b:22015, Zbl 0433.22012

$[\mathrm{H}] \quad \mathrm{R}$. Howe, Classification of irreducible representations of $\mathrm{GL}_{2}(F), F$ a local field, preprint, 1978.

[J-PS-S] H. Jacquet, I. Piatetski-Shapiro and J. Shalika, Rankin-Selberg convolutions, Amer. J. Math., 105(2) (1983), 367-464, MR 85g:11044, Zbl 0525.22018.

[J-Y1] H. Jacquet and Y. Ye, Distinguished representations and quadratic base change for GL(3), Trans. Amer. Math. Soc., 348(3) (1996), 913-939, MR 96h:11041, Zbl 0861.11033.

[PS] I. Piatetski-Shapiro, Complex Representations of $\mathrm{GL}(2, K)$ for Finite Fields K, Contemporary Math., 16, AMS, 1983, MR 84m:20046, Zbl 0513.20026.

[Rod] F. Rodier, Modéle de Whittaker et caract'eres de repr'esentations, Lecture Notes in Mathematics, 466, Springer Verlag, 1974, 151-171, MR 52 \#14165, Zbl 0339.22014.

[S] D. Soudry, The $L$ and $\gamma$ factors for generic representations of $G S p(4, k) \times$ $\mathrm{GL}(2, k)$ over a local non-Archimedean field $k$, Duke Math. J., 51(2) (1984), 355-394, MR 86f:22022, Zbl 0557.12012.

Received February 4, 2002. Partially supported by NSF grant DMS-0070762.

Department of Mathematics

TECHNION

HAIFA 32000

ISRAEL

E-mail address: embaruch@math.technion.ac.il 